\title{
Thermally-Induced Shapes of Unsymmetric Laminates
}

\author{
L. J. B. Peeters, * P. C. Powell AND L. WARneT \\ University of Twente \\ Department of Mechanical Engineering \\ P.O. Box 217, 7500 AE \\ Enschede, The Netherlands \\ (Received August 10, 1994) \\ (Revised April 4, 1995)
}

\begin{abstract}
Models to describe the shapes of unsymmetric laminates produced with a flat mould have been already developed by several researchers. These models are reviewed and on some points theory is extended.

The experimental work done so far to confirm the developed theoretical models is rather limited. This especially applies for angle-ply laminates, on which no experimental work has been reported yet. This paper presents new experimental work that has been conducted both on cross-ply and angle-ply laminates over a wide range of laminate dimensions. The experiments show that the influence of the side length on the curvature is very well predicted both for cross-ply and angle-ply laminates, also the bifurcation point behaviour is clearly observed. Possible causes of differences between theory and experiment are outlined. The theoretical predictions are especially sensitive to the transverse thermal expansion coefficient of the individual layers.
\end{abstract}

\section{INTRODUCTION}

T NSYMMETRIC CONTINUOUS LONG fibre laminates made from advanced polymer matrix unidirectional (UD) plies that are processed hot within flat moulds will develop curvatures upon release after cooling to ambient temperature. The curvatures are a result of unbalanced thermal shrinkage across the laminate thickness. When the curvatures can be well predicted, the production of curved panels from flat moulds can be an attractive technique, especially where prototypes and small quantities of large-area curved panels are required.

According to the Classical Lamination Theory (C.L.T.), hot-cured unsymmetric cross-ply laminates of any size should always develop anticlastic (saddleshaped) curvatures, and unsymmetric angle-ply laminates should develop twisting curvatures. However, large out-of-plane displacements can restrict the develop-

*Author to whom correspondence should be addressed. 
ment of curvatures depending on the size of the plate. A different theoretical approach must be used to predict the cured shape of unsymmetric laminates.

Since the early eighties, several papers concerned with the room-temperature shape of unsymmetric thin laminates have been published. Hyer [1-3] observed that the cured shapes of thin unsymmetric laminates do not conform to the predictions of the classical laminate theory. It was felt by Hyer that the observed anomalies are due to geometric nonlinearities, since the out-of-plane deflections of the laminates were on the order of many laminate thicknesses. Hyer presented a theory based on the minimization of the total potential energy using nonlinear strain-displacement relations to predict the room-temperature shapes of unsymmetric laminates. Approximate solutions were found by using the Rayleigh-Ritz method and from the resulting nonlinear equations, the curvatures were calculated numerically using a Newton-Raphson iteration scheme.

Although the theoretical approach is applicable for all unsymmetric long fibre/polymer laminates, Hyer only looked at cross-ply laminates. Jun and Hong $[4,5]$ also looked at the cured shapes of unsymmetric laminates with arbitrary lay-up angles. Hahn and Hwang [6] presented a different method for calculating the cured shapes of large panels. For an unsymmetric cross-ply laminate they used the C.L.T. and arbitrarily set curvature to be zero. The remaining curvature was obtained through equilibrium conditions. In this paper the different theoretical approaches are compared with each other, with theory which is extended on some points and with finite element analysis.

To date the number of experiments done to validate the developed theory is rather limited $[1,6,7]$. All the reported experimental work has been done on thermosetting based composites; for these laminates the curvatures are influenced by the chemical shrinkage during the cure-cycle as is reported by White and Hahn [8-10]. Chemical shrinkage is not appropriate for thermoplastic matrices. This paper presents new experiments on cross-ply and angle-ply thermoplastic based laminates and possible causes of differences between theory and experiment.

\section{THEORY}

The Classical Lamination Theory (C.L.T.) $[15,16]$ describes the macromechanical behaviour of laminates made from orthotropic plies, with the following major assumptions:

- the displacements are continuous throughout the laminate

- line elements which originally are perpendicular to the middle surface of the plate remain straight and normal to the deformed middle surface and suffer no change in length (Kirchhoffs hypothesis)

- the strain-displacement relationships are linear

- plane stress condition $\left(\sigma_{z}=\sigma_{y z}=\sigma_{x x}=0\right)$

- the laminate is constructed of an anisotropic, homogeneous, linearly-elastic material with properties independent of temperature

In practice, the out-of-plane displacement $w$ of the room temperature shapes of unsymmetric laminated plates processed hot can be many times the plate thick- 
ness. Therefore, the strain-displacement relations cannot be taken linearly anymore. In order to take this into account, the same methodology as Hyer [2] was used. Hyer added nonlinear terms to the strain-displacement relations usually used in the classical lamination theory:

$$
\begin{aligned}
& \epsilon_{x}=\frac{\partial u^{0}}{\partial x}+\frac{1}{2}\left(\frac{\partial w}{\partial x}\right)^{2}-z \frac{\partial^{2} w}{\partial x^{2}}=\epsilon_{x}^{0}-z \frac{\partial^{2} w}{\partial x^{2}} \\
& \epsilon_{y}=\frac{\partial \nu^{0}}{\partial y}+\frac{1}{2}\left(\frac{\partial w}{\partial y}\right)^{2}-z \frac{\partial^{2} w}{\partial y^{2}}=\epsilon_{y}^{0}-z \frac{\partial^{2} w}{\partial y^{2}} \\
& \epsilon_{x y}=\frac{1}{2}\left(\frac{\partial u^{0}}{\partial y}+\frac{\partial v^{0}}{\partial x}+\frac{\partial w}{\partial x} \frac{\partial w}{\partial y}\right)-z \frac{\partial^{2} w}{\partial x \partial y}=\epsilon_{x y}^{0}-z \frac{\partial^{2} w}{\partial x \partial y}
\end{aligned}
$$

The index 0 is used for the laminate midplane, i.e., $z=0$.

The theory [2] is based on the principle of virtual work (in this case this means minimization of the total potential energy). Since no external load is applied, the total potential energy of a laminate, including thermal effects, is given by [18]:

$$
W=\int_{V o l}\left(\frac{1}{2} Q_{i j}^{\prime} \epsilon_{i} \epsilon_{j}-\beta_{i} \epsilon_{j} \Delta T\right) d V o l
$$

where the $Q_{i j}^{i}$ are the reduced transformed stiffness coefficients, the $\beta_{i}$ are coefficients related to the elastic and thermal properties of the material $\left(\beta=Q_{i j}^{\prime} \alpha_{j}\right.$ with $\alpha_{j}$ the thermal expansion coefficients) and $\Delta T$ is the temperature change.

In order to obtain approximate solutions the Rayleigh-Ritz method was used. This means that the displacement field is approximated by functions which contain a finite number of independent coefficients. The next step is then to define the displacement field. This was done for cross-ply laminates by Hyer [3] and Jun and Hong [4].

\subsection{Cross-Ply [0/90] Laminates}

Cross-ply laminates can cure into a saddle shape or into one of two possible cylindrical shapes. Both Hyer [2,3] and Jun and Hong [4,5] used a third order approximation of these shapes:

$$
w(x, y)=\frac{1}{2}\left(a x^{2}+b y^{2}\right)+O\left(x^{4}, y^{4}\right)
$$

Both $a$ and $b$ are to-be-determined constants. $O\left(x^{4}, y^{4}\right)$ indicates terms of a four or higher order in $x$ or $y$, which are not taken into consideration. It happens here that the terms in the third order are zero.

The shapes, which to a certain extent can be described by the equation for the out-of-plane displacements (3), are shown in Figure 1: (a) shows the shape at 


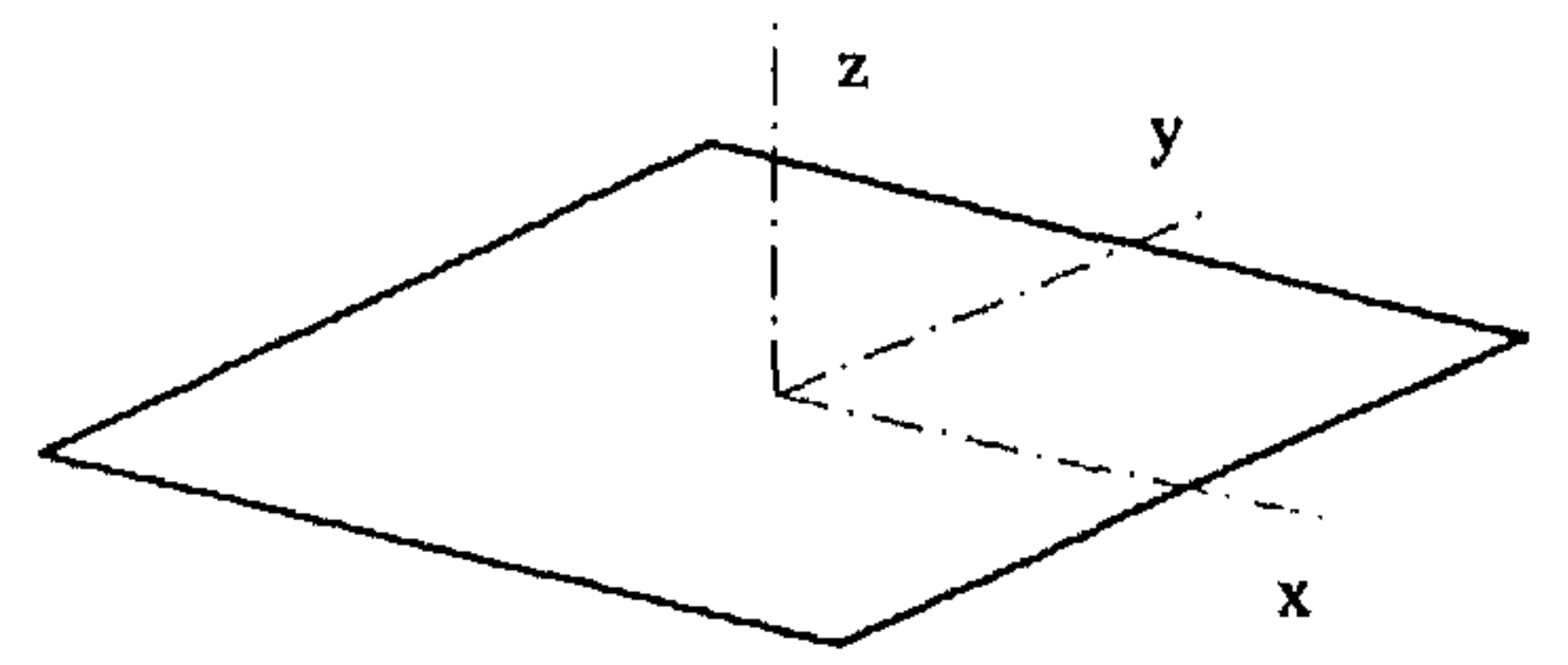

(a)

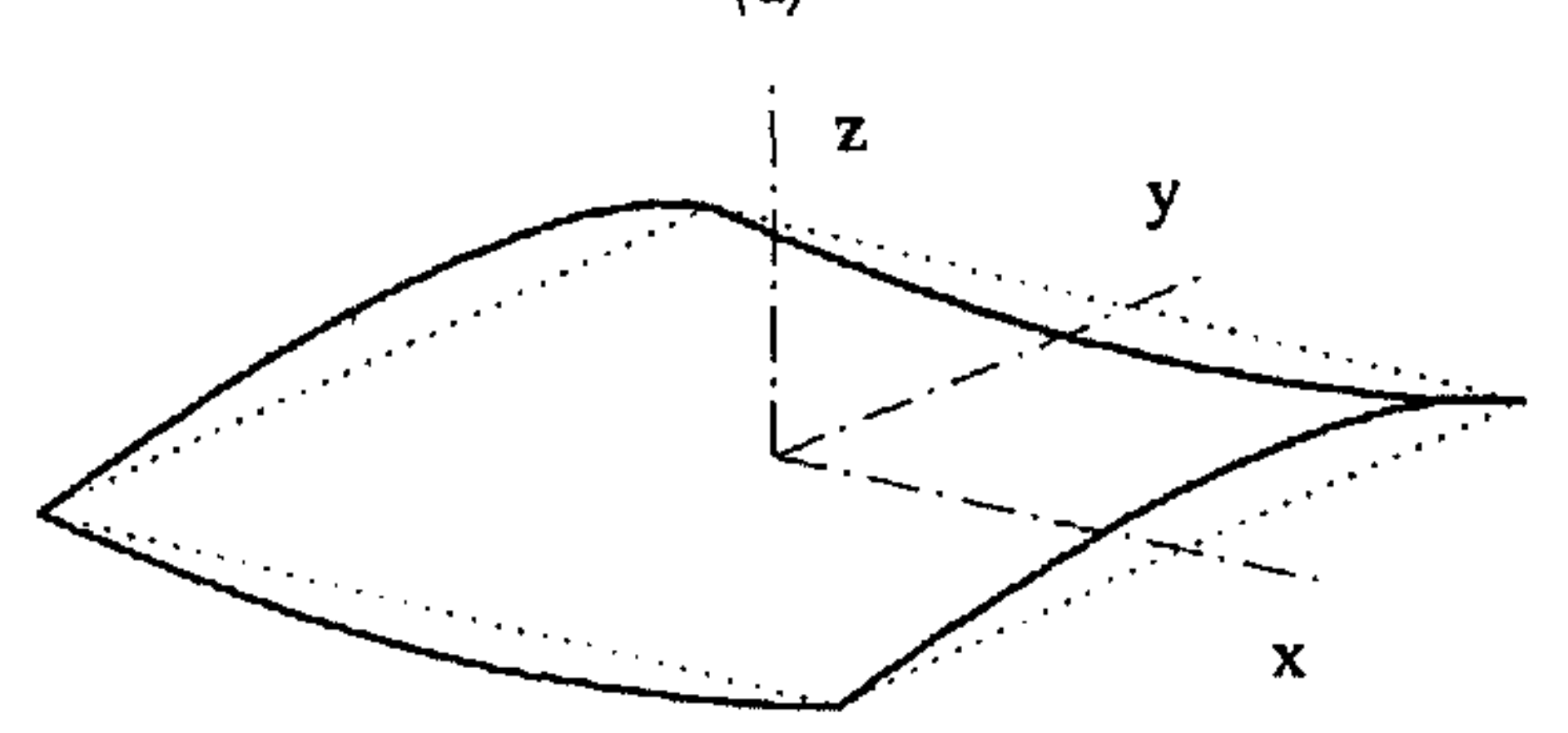

(b)

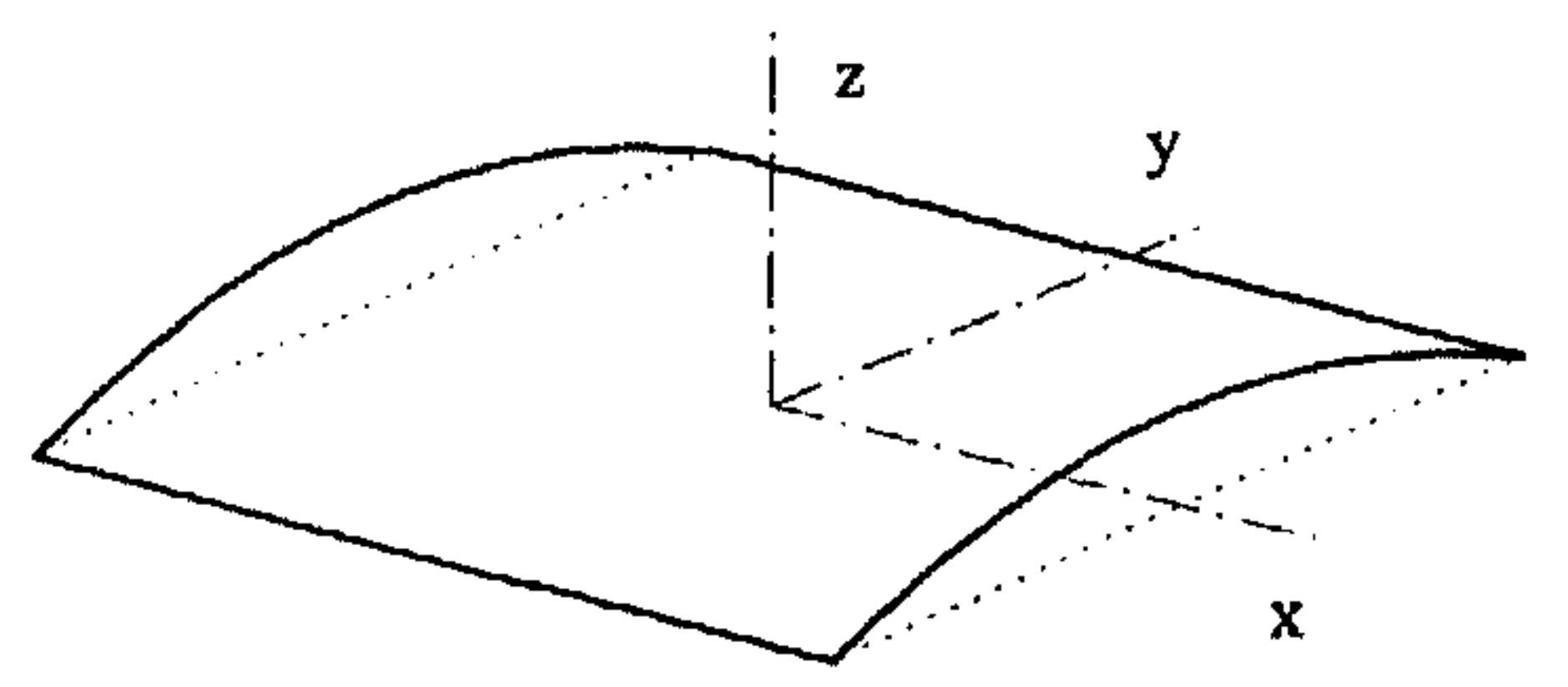

(c)

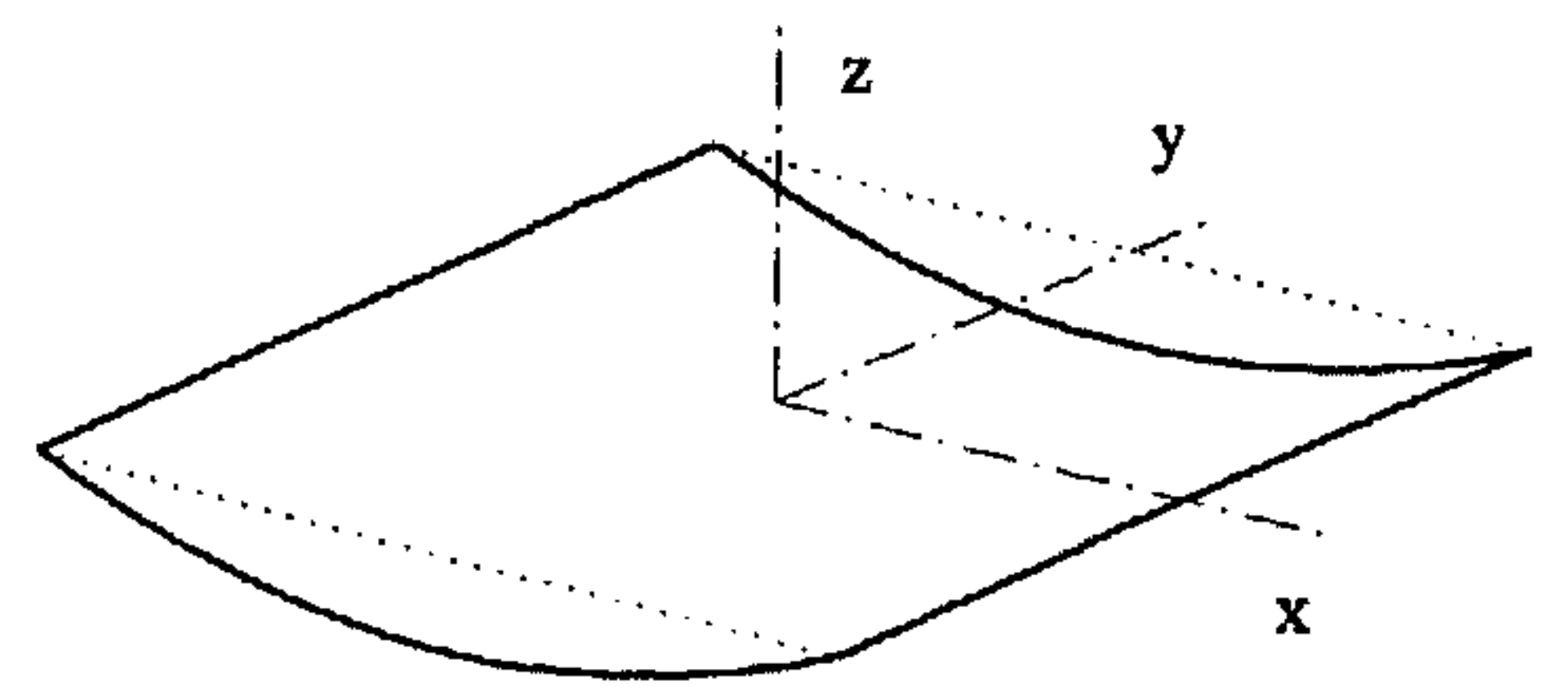

(d)

Figure 1. Room temperature shapes of cross-ply laminates.

stress-free temperature, (b) shows the saddle shape predicted by the classical lamination theory $(a=-b)$ and (c) and (d) show the two stable cylindrical shapes usually obtained in practice $(a=0$ or $b=0)$. From one cylindrical shape, the other one can be obtained by a snap-through action.

A general form of in-plane displacement is chosen, as in Timoshenko [14]:

$$
u^{0}(x, y)=x\left(a_{1}+a_{2} x^{2}+a_{3} y^{2}\right) \quad v^{0}(x, y)=y\left(b_{1}+b_{2} y^{2}+b_{3} x^{2}\right)
$$


The following assumptions help reduce the amount of unknowns:

- $u^{0}(0, y)=0 ; v^{0}(x, 0)=0$

- $\epsilon_{x}^{0}$ independent of $x ; \epsilon_{y}^{0}$ independent of $y, \epsilon_{x y}^{0}=0$

Working out those assumptions in the displacement field and substituting the result in the strain-displacement relations (l) gives the strain field according to Hyer:

$$
\epsilon_{x}=a_{1}-\frac{a b y^{2}}{4}-a z \quad \epsilon_{y}=b_{1}-\frac{a b x^{2}}{4}-b z \quad \epsilon_{x y}=0
$$

The strain field equations are then substituted into the developed version of the total potential energy (2) (see the classical lamination theory [12] for the expressions of the $Q_{i j}^{\prime}$ ).

$$
\begin{aligned}
W & =\int_{x} \int_{y} \int_{z}\left[\frac{1}{2} Q_{11}^{\prime} \epsilon_{x}^{2}+Q_{12}^{\prime} \epsilon_{x} \epsilon_{y}+\frac{1}{2} Q_{22}^{\prime} \epsilon_{y}^{2}+2 Q_{66}^{\prime} \epsilon_{x y}^{2}\right. \\
& -\left(Q^{\prime}{ }_{11} \alpha_{x}^{\prime}+Q_{12}^{\prime} \alpha_{y}^{\prime}\right) \epsilon_{x} \Delta T-\left(Q_{22}^{\prime} \alpha_{y}^{\prime}+Q_{12}^{\prime} \alpha_{x}^{\prime}\right) \epsilon_{y} \Delta T \\
& \left.-2 Q_{66}^{\prime} \alpha_{x y}^{\prime} \epsilon_{x y} \Delta T\right] d x d y d z
\end{aligned}
$$

Minimising the total potential energy means:

$$
\partial W=\sum_{i=1}^{n}\left(\frac{\partial W}{\partial a_{i}}\right) \partial a_{i}=0
$$

The $a_{i}$ are the unknown Ritz-coefficients, $n$ the number of unknowns ( 4 for Hyer: $a, a_{i}, b$ and $b_{i}$ ). The problem to be solved becomes the following:

$$
\frac{\partial W}{\partial a_{i}}\left(a_{i}, L\right)=0 ; \quad \text { for } i=1, \ldots, n ; L=\text { constant }
$$

This is a system of $n$ nonlinear algebraic equations. A Newton-Raphson iteration procedure can then be used to calculate the curvatures numerically. For a stable solution $\partial^{2} W$ has to be positive and definite.

A typical square cross-ply dimension-curvature relationship, which is obtained by applying the described theory, is shown in Figure 2. It shows on the first part a single branch $A B$ which describes the anticlastic shape $\left(x_{x}=-x_{y}\right)$. The critical length for this shape to be stable is a so-called bifurcation-limit point (B). Branches $B C$ and $B D$ represent, respectively, stable cylinders with axes in the $x$ and $y$-direction. Because the diagrams (for $\varkappa_{x}$ and $x_{y}$ ) are symmetric with respect to the horizontal axis, one diagram can be used to present the dimensioncurvature relationship, without loss of information. 

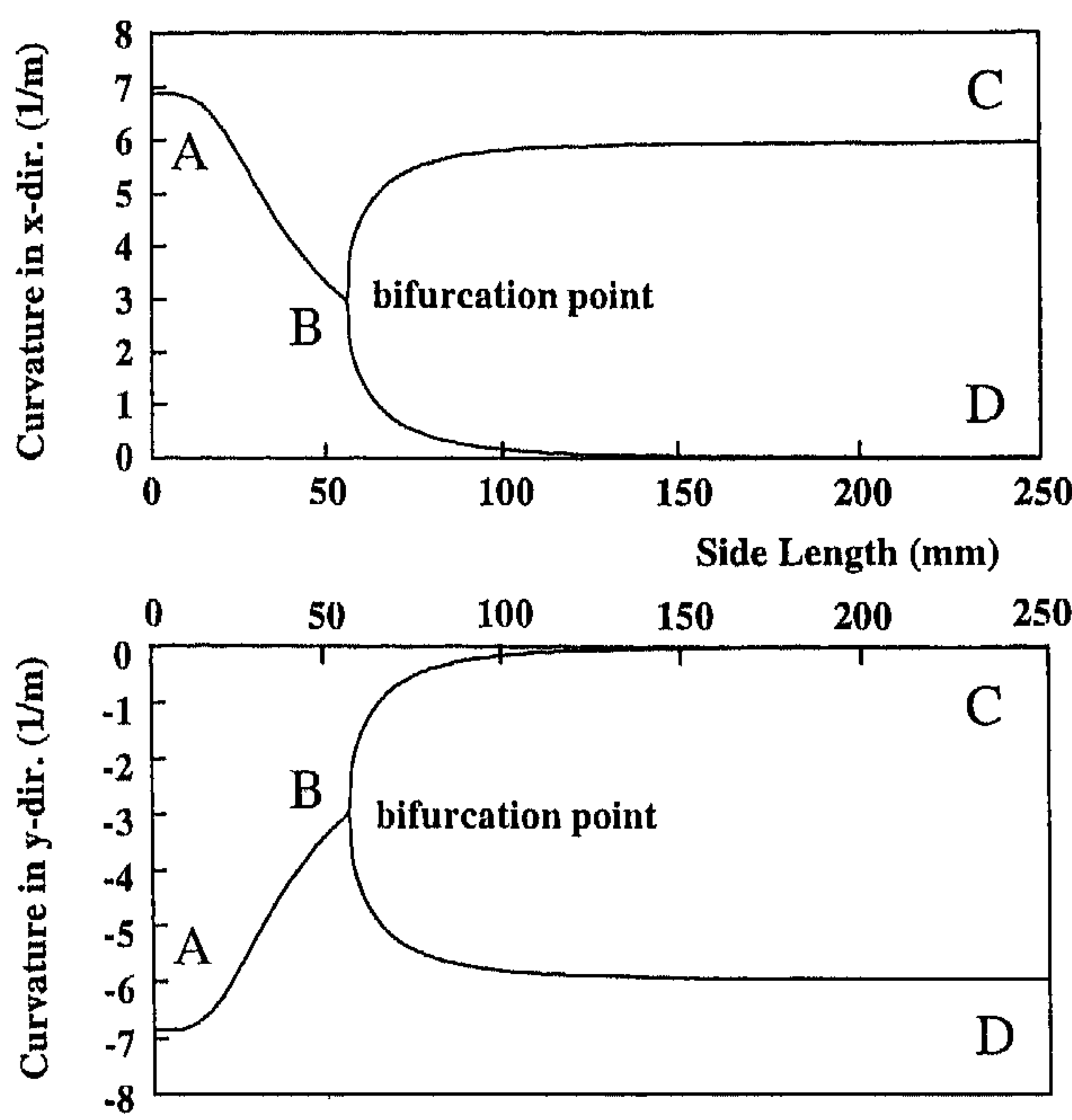

Figure 2. Typical cross-ply dimension-curvature relationship.

Jun and Hong [4] correctly argued that the thermal residual in-plane shear strain should not be neglected. If the shape of a plate is not perfectly cylindrical shear strain is present, because the term $\partial w / \partial x \partial w / \partial y$ in Equation (1) is not zero anymore. Even for very large panels the shapes are not perfectly cylindrical because of free-edge effects, therefore shear strains will always be present in unsymmetric cross-ply laminates. The largest shear strains are found near the corners as it is shown in Figure 3. Figure 3 shows the shear strain distribution of a square saddle-shaped cross-ply laminate, established by use of finite element analysis. The numbers 1-8 indicate iso-strain profiles and the cross-hatches show the positions of the element nodes. In terms of side length the laminate is near the bifurcation length and because of symmetry conditions only one quarter of the laminate was modelled. Locally the shear strain can even be larger than the normal in-plane strains.

To take shear strain into account Jun and Hong approximated the displacement field by the following functions, involving 6 unknowns (instead of 4 for Hyer's):

$$
\begin{gathered}
u^{0}(x, y)=x\left(a_{1}-\frac{a^{2}}{6} x^{2}+a_{3} y^{2}\right) v^{0}(x, y)=y\left(b_{1}-\frac{b^{2}}{6} y^{2}+b_{3} x^{2}\right) \\
w(x, y)=\frac{1}{2}\left(a x^{2}+b y^{2}\right)
\end{gathered}
$$




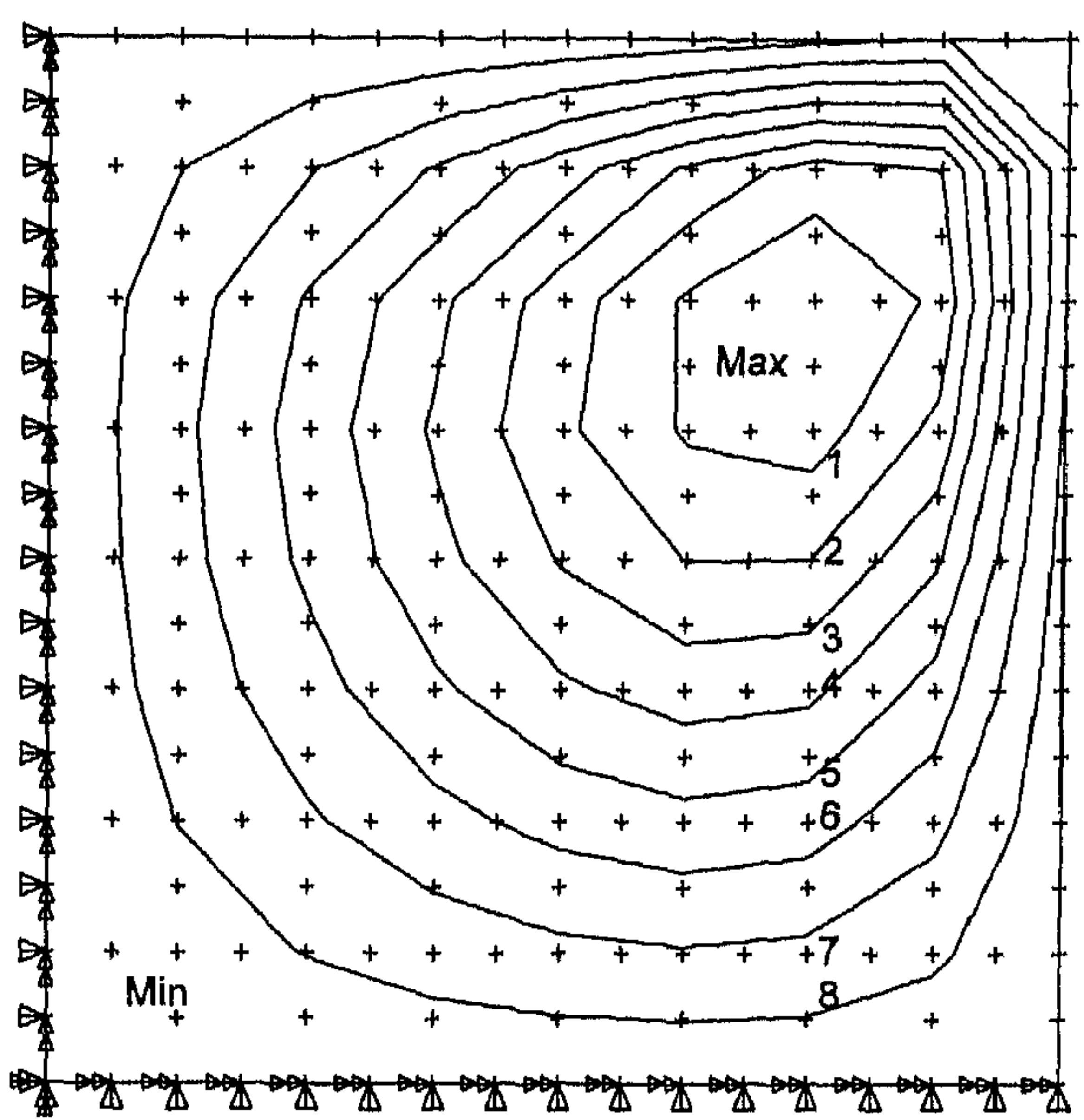

Flgure 3. Shear strain distribution in a quarter of an unsymmetric cross-ply laminate.

Substituting these displacement functions in the strain-displacement relations (1) gives the strain field according to Jun and Hong:

$\epsilon_{x}=a_{1}+a_{3} y^{2}-a z \quad \epsilon_{y}=b_{1}+b_{3} x^{2}-b z \quad \epsilon_{x y}=\left(a_{3}+b_{3}+a b / 2\right) x y$

In their paper Jun and Hong [4] showed that in-plane shear strain has a significant influence on the position of the bifurcation point and the curvatures in the vicinity of the bifurcation point. In cases where the side length (only square laminates are considered) is either very small or large compared with the length at the bifurcation point, the thermal residual shear strain is small and can be neglected. This is clearly shown in Figure 4, where the methods of Hyer and Jun and Hong are compared for an unsymmetric two layer cross-ply laminate. However, from a designer point of view, large area panels are more interesting (side length $\rightarrow$ length at the bifurcation point). Therefore, for panels which would be used in practice, the methods of Hyer and Jun and Hong give similar results.

An analytical equation for calculating the asymptotic value for the cylindrical deformations, derived from the classical lamination theory, is presented by Hahn and Hwang [6]. By setting one of the curvatures $\left(x_{y}\right)$ to be zero they obtained for the curvature of a (cylindrical) cross-ply laminate:

$$
\varkappa_{c}=\frac{\left(A_{11} A_{22}+A_{12}^{2}\right) M_{x}^{x}-B_{11}\left(A_{22}-A_{12}\right) N_{x}^{T}}{D_{11}\left(A_{11} A_{22}-A_{12}^{2}\right)-B_{11}^{2} A_{22}}
$$


where $N_{x}^{T}$ is the thermal force resultant per unit width along the $x$-direction, $M_{x}^{T}$ is the thermal moment resultant per unit width along the $x$-direction and $A_{i j}, B_{i j}$ and $D_{i j}$ are the laminate stiffnesses $[15,16]$.

For large panels the curvature $\varkappa_{c}$ resulting from Equation (11) is exactly similar to the asymptotic value of the curvature obtained by using the method of Hyer or Jun and Hong. Thus, for predicting the curvature of large panels, it is relatively simple to use Equation (11) instead of the approach of Hyer or Jun and Hong.

As previously mentioned, both Hyer and Jun and Hong used a third order approximation of the saddle shape and the cylindrical shapes as the out-of-plane displacement field. This approximation is acceptable for small plate sizes, but for larger plates the deviations are quite substantial, e.g., for a plate which curves into half a cylinder the deviation of the out-of-plane displacement can be as large as $50 \%$. For a cross section of a cylindrical shape, which is a circle segment, this is shown in Figure 5. Near the edges, the order 5 approximation and especially the order 3 approximation result in a bad description of the out-of-plane displacement field $w(x)$.

It was felt that using a better (higher order) approximation of a circle could influence the calculated curvatures. Therefore a higher (5th) order approximation of a circle was used in the equation for the out-of-plane displacement field:

$$
w(x, y)=\frac{1}{2} a x^{2}+\frac{1}{8} a^{3} x^{4}+\frac{1}{2} b y^{2}+\frac{1}{8} b^{3} y^{4}+O\left(x^{6}, y^{6}\right)
$$

The terms in the fifth order happen to be zero and $O\left(x^{6}, y^{6}\right)$ indicates terms of a sixth or higher order in $x$ or $y$, which are not taken into consideration.

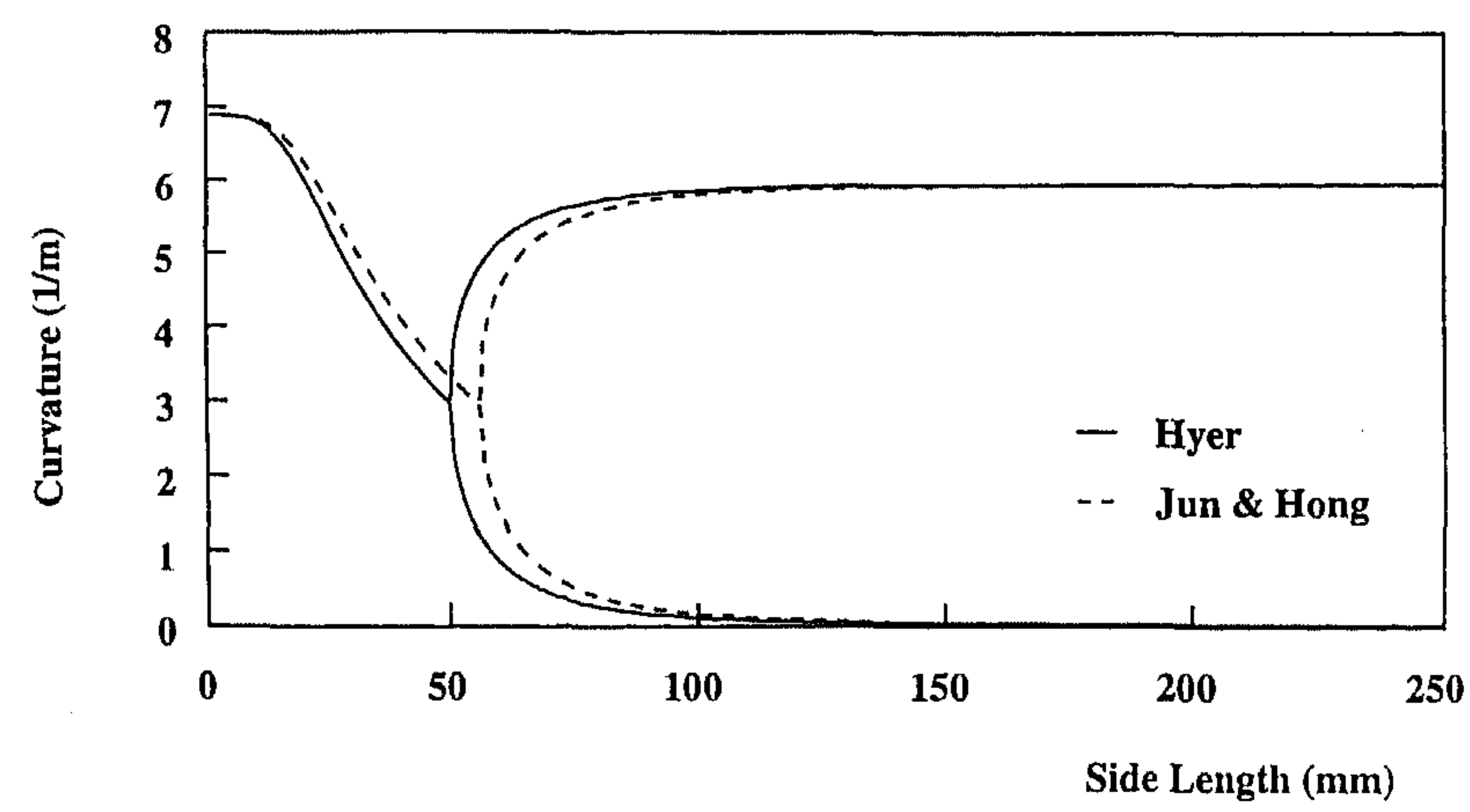

Figure 4. Method of Hyer compared with method of Jun \& Hong for a [0/90]T glass/PEI laminate (ply thickness $h_{p}=0.36 \mathrm{~mm}$ ). 


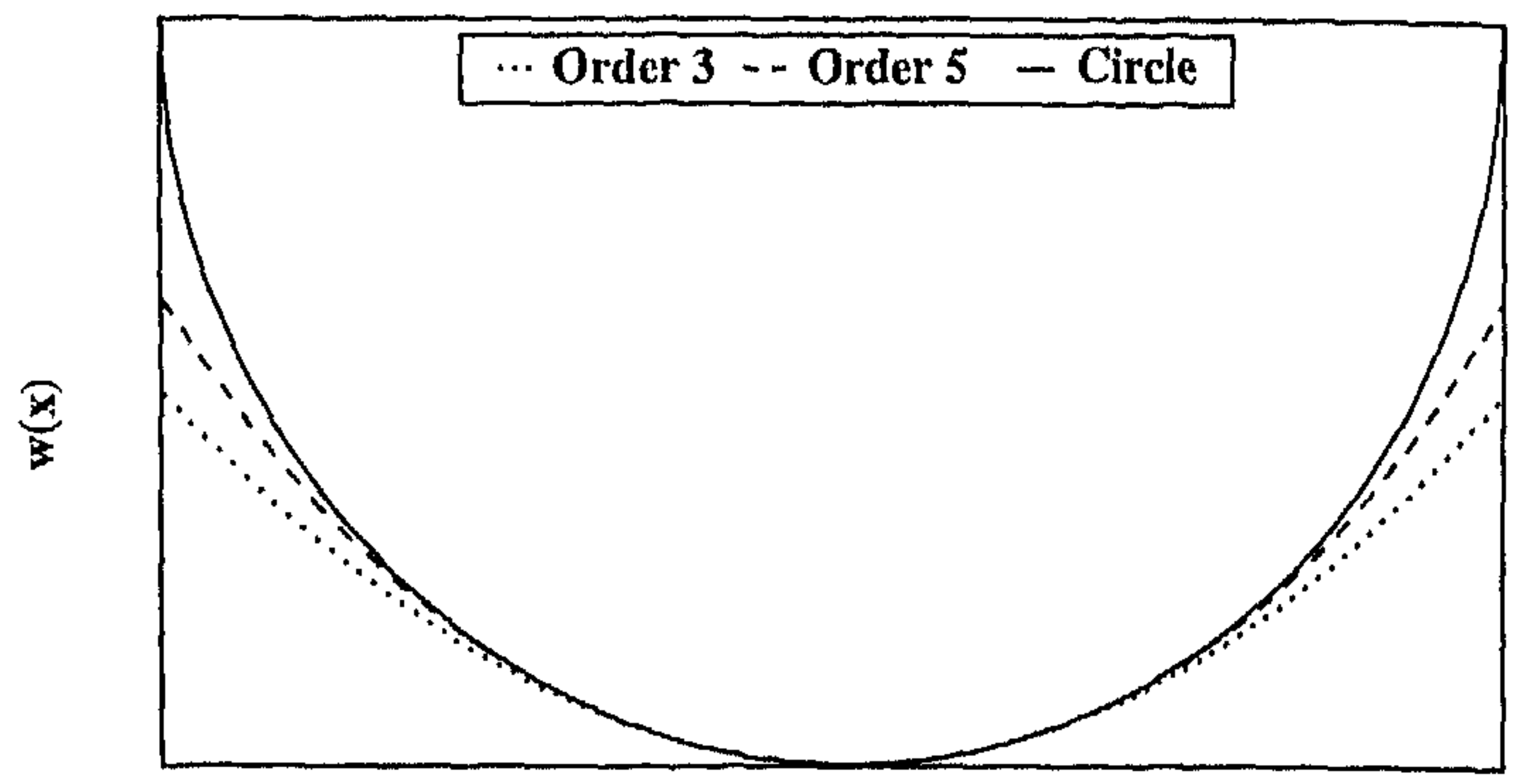

$\mathbf{x}$

Flgure 5. Approximations of a circle.

Applying the assumptions $\epsilon_{x}^{0}$ independent of $x$ and $\epsilon_{y}^{0}$ independent of $y$, results in the following in-plane displacement functions:

$$
\begin{aligned}
& u^{0}(x, y)=x\left(a_{1}-\frac{a^{2}}{6} x^{2}-\frac{a^{4}}{10} x^{4}-\frac{a^{6}}{56} x^{6}+a_{3} y^{2}\right) \\
& v^{0}(x, y)=y\left(b_{1}-\frac{b^{2}}{6} y^{2}-\frac{b^{4}}{10} y^{4}-\frac{b^{6}}{56} y^{6}+b_{3} x^{2}\right)
\end{aligned}
$$

In Figure 6 the curvatures calculated by using the displacement functions (12) and (13) are compared with the curvatures calculated using the displacement functions (9) suggested by Jun and Hong [4]. The differences are negligible; therefore, it is concluded that the third order approximation of a circle for the out-of-plane displacement field is accurate enough to obtain reliable values for the curvatures. There is no need to use a better approximation of a circle.

Finally in Figure 7 the curvatures calculated with the method described above are compared with curvatures calculated with a finite element method using layered shell elements. Nonlinear thermal analyses were performed with the ANSYS finite element program. To obtain the finite element solutions only one quarter of the plate needs to be modelled, because of symmetry (see also Figure 3). A mesh was chosen such that refining the mesh by a factor 2 resulted in a curvature change of less than 1 percent.

In both methods solutions are obtained through application of the principle of minimum potential energy. This means that the theory using displacement functions (9) should give more or less similar results than the results obtained with the finite element method. Figure 7 shows that this is the case, therefore, it is concluded that with the method used by Jun and Hong reliable results can be obtained for the cured shapes of unsymmetric cross-ply laminates - at least when 


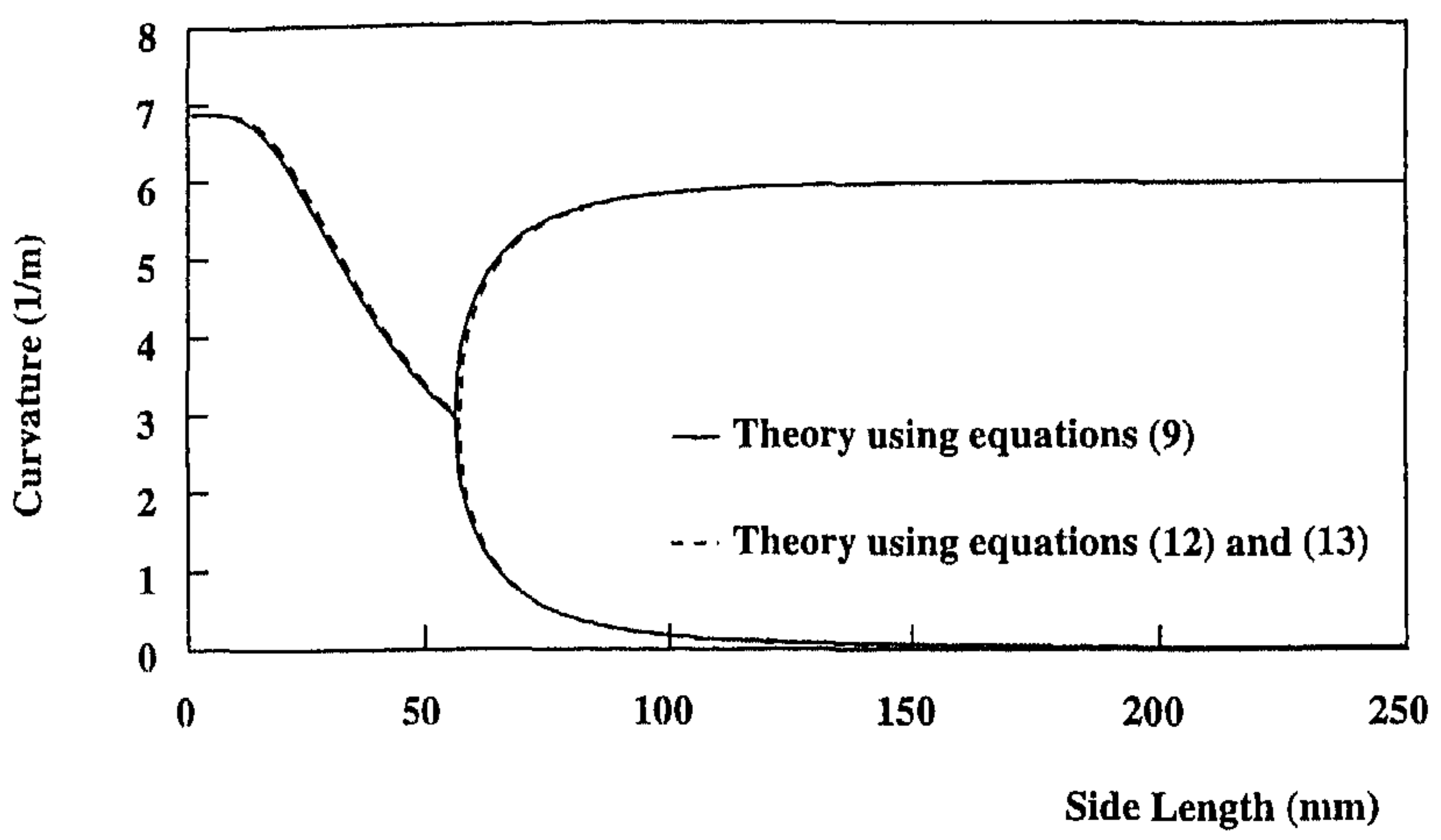

Flgure 6. Theory using modifled displacement functions (12) and (13) compared with theory using displacement functions (9) for a [0/90] glass/PEl laminate (ply thickness $h_{p}=$ $0.36 \mathrm{~mm}$ ).

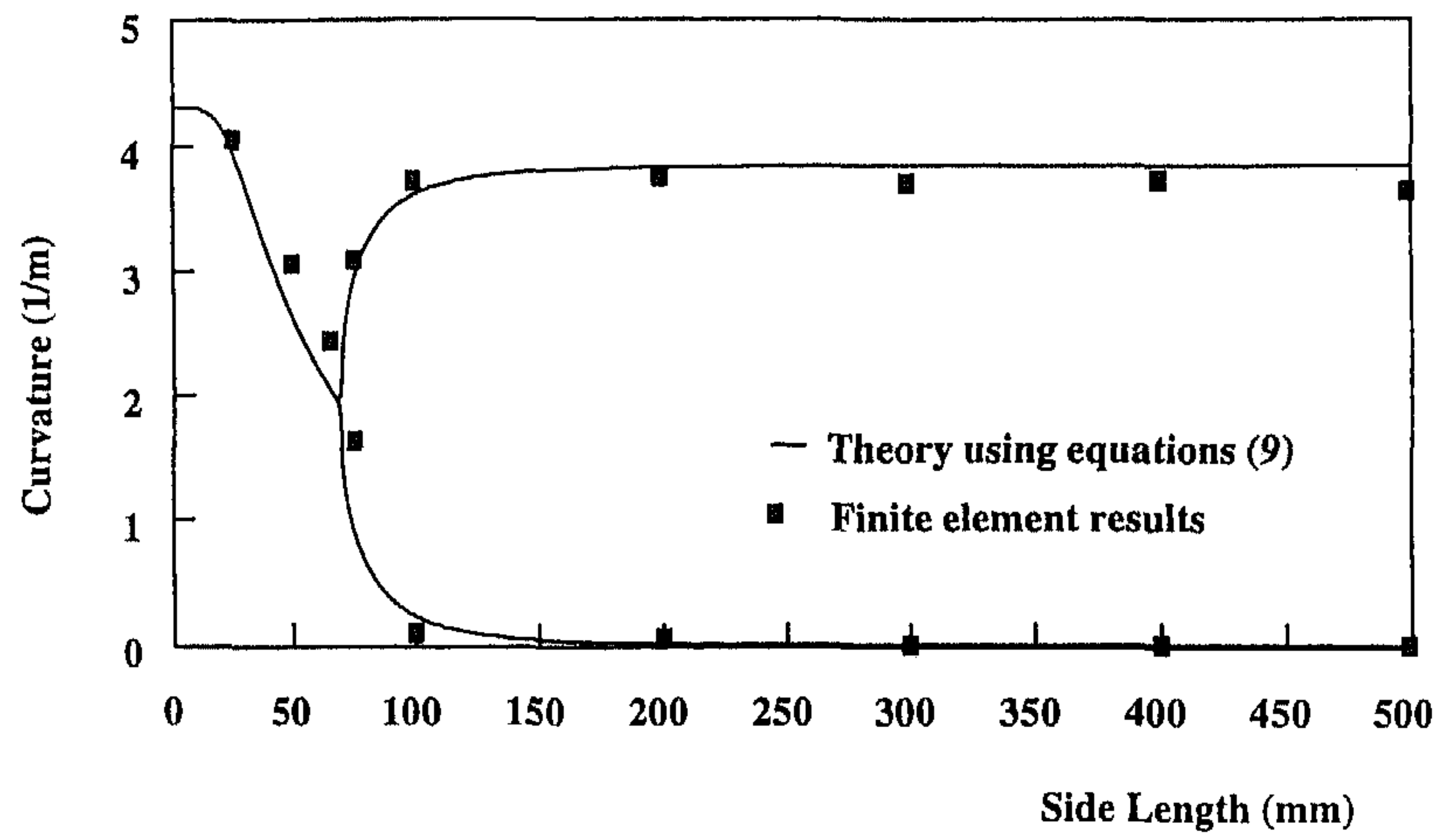

Figure 7. Theory using displacement functions (9) compared with finite element results for a [0/90] glass/PEl laminate (ply thickness $h_{p}=0.36 \mathrm{~mm}$; different fibre volume fraction than in figures presented so far). 
the input parameters (mechanical and thermal properties and the temperature difference between the stress-free temperature and the room temperature) are correct.

\subsection{Angle-Ply $[+\theta /-\theta]$ Laminates}

Jun and Hong [5] presented a method that calculates the curvatures of unsymmetric laminates with arbitrary lay-up angles. They described curvatures in the
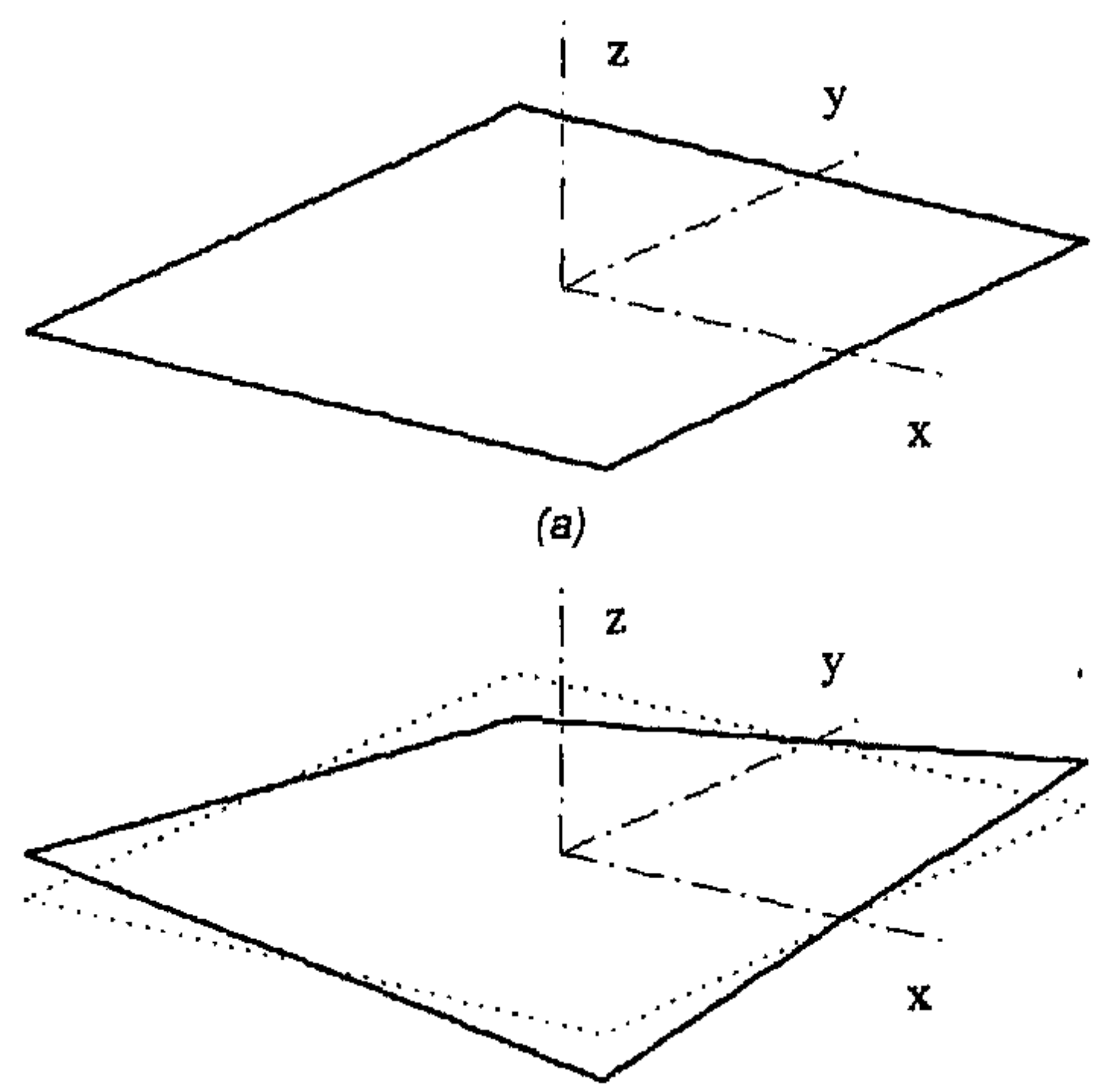

(b)

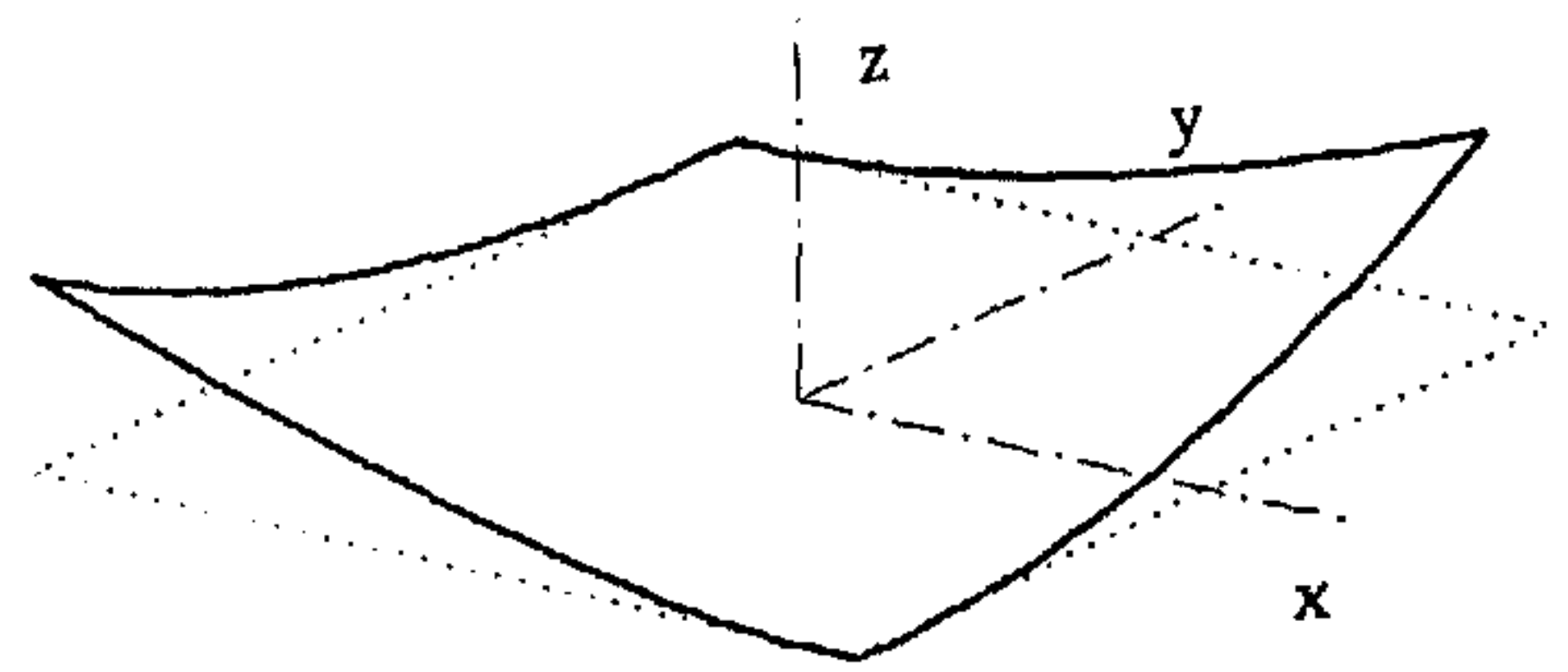

(c)

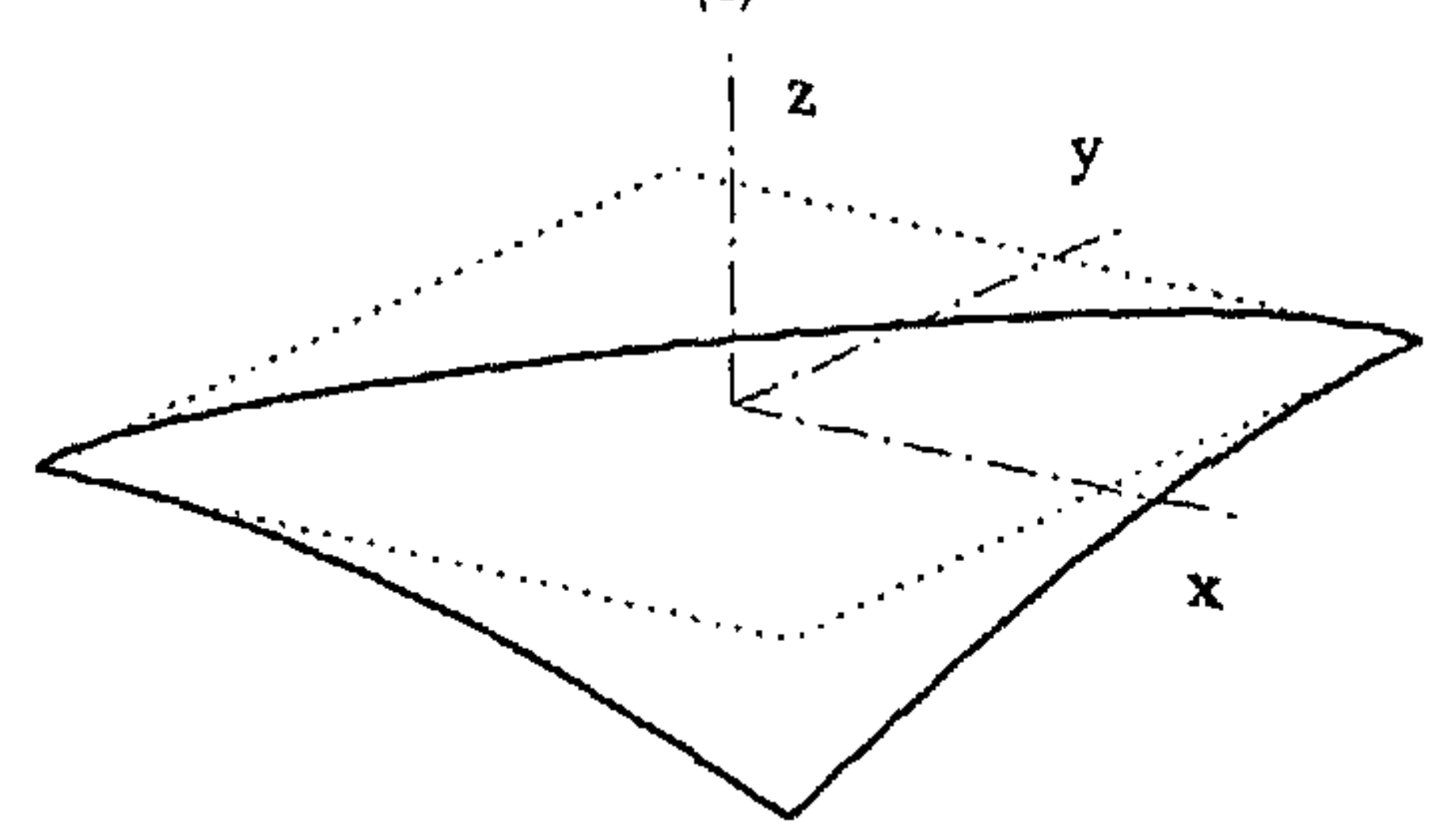

(d)

Figure 8. Room temperature shapes of angle-ply laminates. 


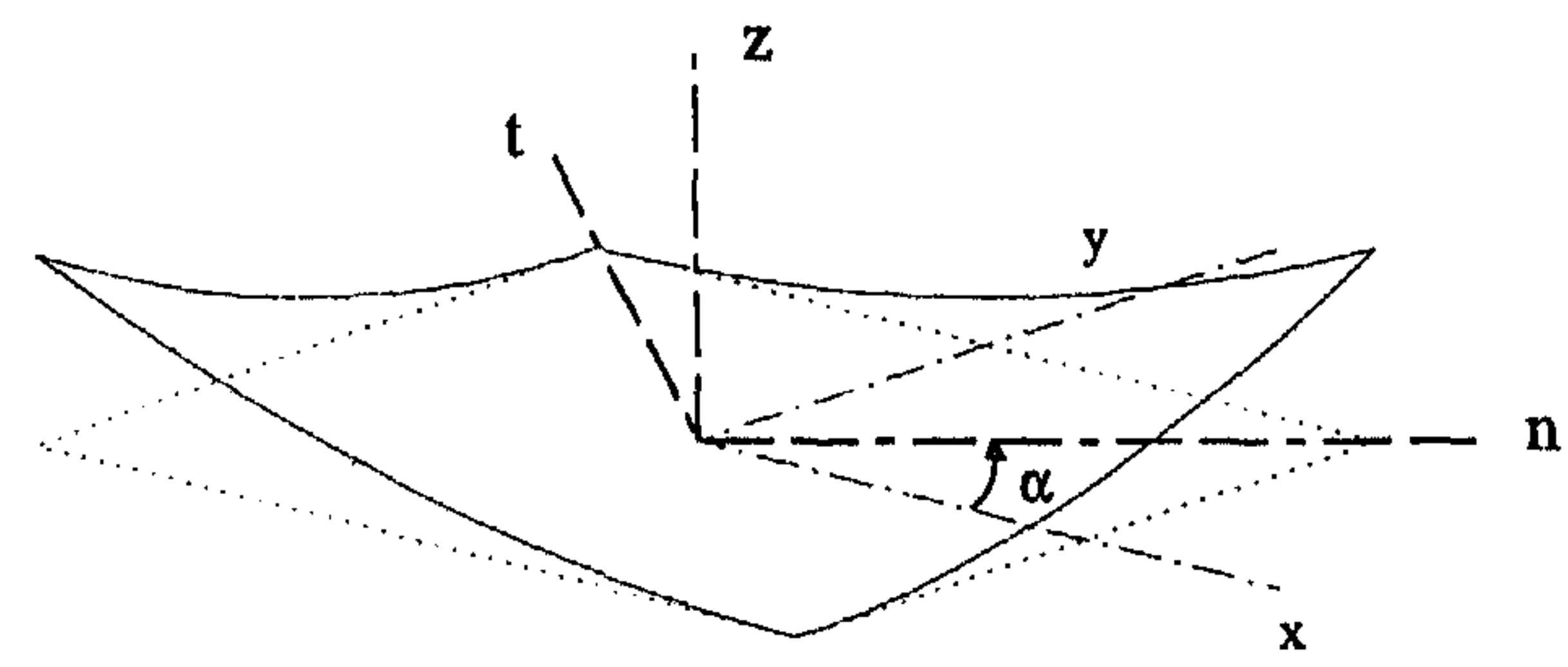

Figure 9. Principal angle of curvature coordinate system n-t-z.

natural body coordinate system. Figure 8 shows the cured shapes of angle-plies at room temperature. From an initially flat lay-up (a), the following shapes can be obtained: (b) shows the purely twisted shape predicted by classical lamination theory and (c) and (d) show the two stable cylindrical shapes usually obtained in practice.

The principal angle of curvature $\alpha$, i.e., the rotation required to get from the laminate coordinate system to the principal curvature system is given by Hyer [1], with $\kappa_{x}, x_{y}$ and $x_{x y}$ based on the classical lamination theory:

$$
\cot 2 \alpha=\frac{\varkappa_{x}-\varkappa_{y}}{2 \varkappa_{x y}}
$$

The classical lamination theory shows $x_{x}=x_{y}$. The principal angle of curvature for $[+\theta l-\theta]$ laminates predicted by this theory is thus $45^{\circ}$.

Observations have shown that for square angle-ply $[+\theta /-\theta]$ laminates, the principal angle of curvature is constant with the side length. Therefore, it is relatively straight forward to work in the principal curvature coordinate system $n-t-z$ shown in Figure 9.

To predict the cured shapes of angle-ply laminates, a similar approach as for the cross-ply laminates will be used. In the case of an angle-ply laminate, shear strain will be present all over the plate, which was taken into account by Jun and Hong [5]. However, they had no constant term in the equation for the shear strain [Equation (10): $\epsilon_{x y}=0$ at $x=0$ and $y=0$ ]. Finite element calculation shows that there is also a certain amount of shear strain in the centre of the plate. Therefore, some terms were added to the out-of-plane displacement field used by Jun and Hong. Using the 3rd order approximations of the resulting shapes this gives the following equation:

$$
w(n, t)=\frac{1}{2} a n^{2}+c n+\frac{1}{2} b t^{2}+d t+O\left(n^{4}, t^{4}\right)
$$

$O\left(n^{4}, t^{4}\right)$ indicates again terms of a fourth or higher order in $n$ or $t$, which are not taken into consideration.

It is still assumed [14] that the longitudinal strains in the midplane, $\epsilon_{n}^{0}$ and $\epsilon_{t}^{0}$, 
are independent of $n$ and of $t$, respectively, and that $u^{0}(0, t)=v^{0}(n, 0)=0$. These conditions lead to the following in-plane displacement functions:

$$
\begin{aligned}
& u^{0}(n, t)=n\left(a_{1}-\frac{c^{2}}{2}-\frac{a c}{2} n-\frac{a^{2}}{6} n^{2}+a_{3} t^{2}\right) \\
& v^{0}(n, t)=t\left(b_{1}-\frac{d^{2}}{2}-\frac{b d}{2} t-\frac{b^{2}}{6} t^{2}+b_{3} n^{2}\right)
\end{aligned}
$$

Using the strain-displacement relationships with geometric nonlinearity (1), strain functions are of the form:

$$
\begin{gathered}
\epsilon_{n}=a_{1}+a_{3} t^{2}-a z \quad \epsilon_{t}=b_{1}+b_{3} n^{2}-b z \\
\epsilon_{x y}=a_{3} n t+b_{3} n t+\frac{1}{2}(c+a n)(d+b t)
\end{gathered}
$$

Again these strain functions are substituted into the developed version of the total potential energy (2). This developed version is not identical to that of crossply laminates (6); $Q_{16}^{\prime}, Q_{26}^{\prime}$ and $\alpha_{x y}$ (or in this case $\alpha_{n t}$ ) are no longer zero. Therefore, the expression for the total potential energy for angle-ply laminates is of the form:

$$
\begin{aligned}
W & =\int_{n} \int_{t} \int_{z}\left[\frac{1}{2} Q_{11}^{\prime} \epsilon_{n}^{2}+Q_{12}^{\prime} \epsilon_{n} \epsilon_{t}+\frac{1}{2} Q_{22}^{\prime} \epsilon_{t}^{2}+2 Q_{16}^{\prime} \epsilon_{n} \epsilon_{n t}\right. \\
& +2 Q_{{ }_{2}}^{\prime} \epsilon_{t} \epsilon_{n t}+2 Q_{66}^{\prime} \epsilon_{n t}^{2}-\left(Q_{11}^{\prime} \alpha_{n}^{\prime}+Q_{12}^{\prime} \alpha_{t}^{\prime}+2 Q_{16}^{\prime} \alpha_{n t}^{\prime}\right) \epsilon_{n} \Delta T \\
& -\left(Q_{12}^{\prime} \alpha_{n}^{\prime}+Q_{22}^{\prime} \alpha_{t}^{\prime}+2 Q_{26}^{\prime} \alpha_{n t}^{\prime}\right) \epsilon_{t} \Delta T \\
& \left.-2\left(Q_{16}^{\prime} \alpha_{n}^{\prime}+Q_{26}^{\prime} \alpha_{t}^{\prime}+2 Q_{66}^{\prime} \alpha_{n t}^{\prime}\right) \epsilon_{n t} \Delta T\right] d n d t d z
\end{aligned}
$$

Minimising this potential energy results in a system of eight nonlinear algebraic equations, which can be solved in the same way as described for the crossply laminates. Figure 10 compares theoretical predictions of curvature against side length for a glass/PEI angle-ply laminate using (a): displacement functions presented above (15) and (16) and (b): displacement functions suggested by Jun and Hong [5]. The influence of the modifications in the displacement functions on the position of the bifurcation point is evident in Figure 10.

More or less in the same way as is shown for the cross-ply laminates, an analytical equation for calculating the asymptotic value for the cylindrical deformation [(c) and (d) in Figure 8] can be derived from the classical lamination theory. First the stress-strain relations must be transformed to the principal curvature coor- 


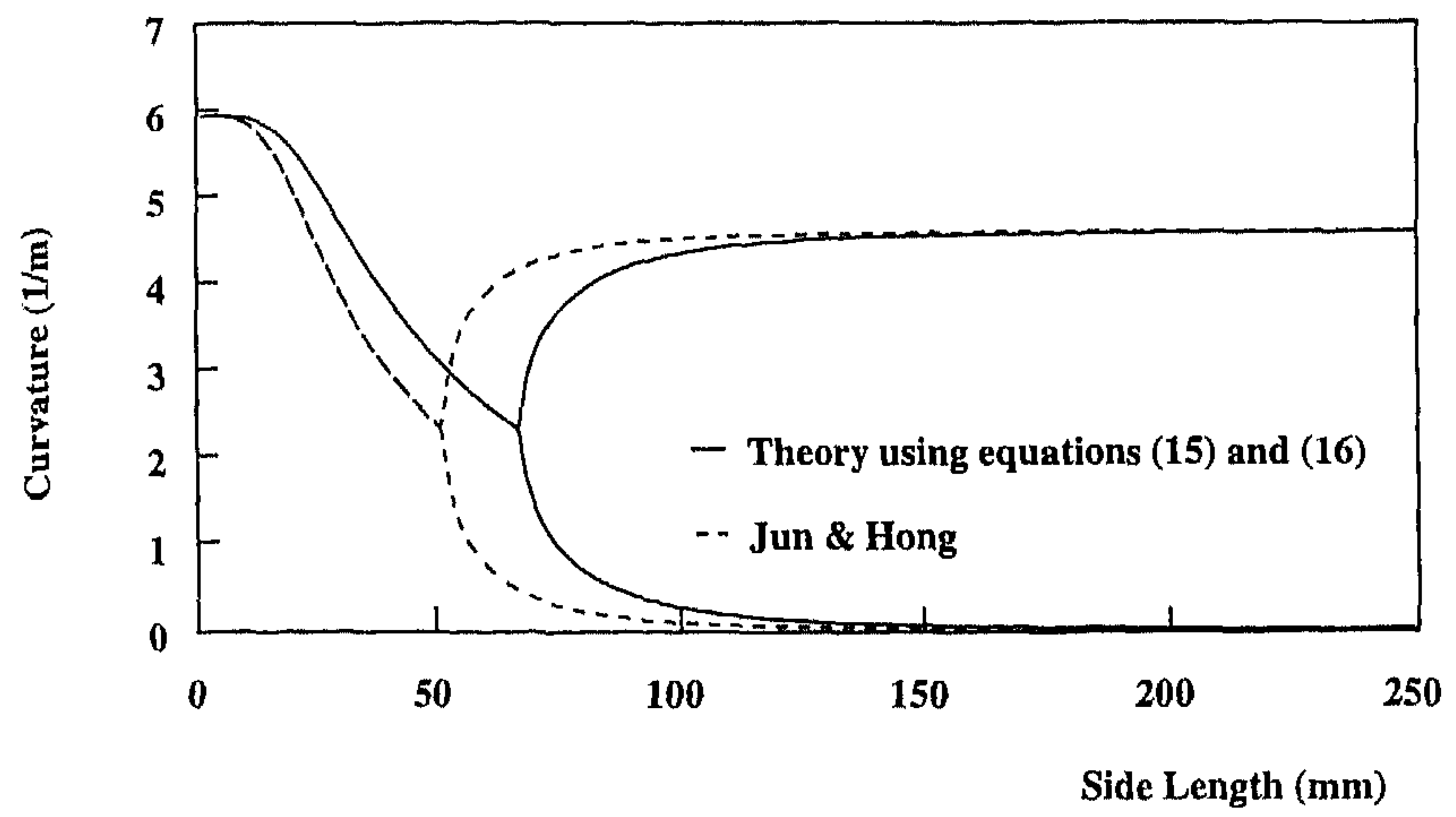

Figure 10. Theory using displacement functions (15) and (16) compared with theory using displacement functions suggested by Jun and Hong [5] for a [30/-30]T glass/PEI laminate (ply thickness $\mathrm{h}_{\mathrm{p}}=0.36 \mathrm{~mm}$ ).

dinate system $n-t-z$. The study of a square angle-ply $[\theta /-\theta]$ laminate will then turn into the study of a square angle-ply $[-\theta+\alpha / \theta+\alpha]$ laminate in the $n-t-z$ coordinate system, where $\alpha$ is defined in Figure 9. Secondly the curvature for the cylindrical deformation can be obtained by setting one of the curvatures $x_{n}$ or $x_{t}$ and the twisting curvature $x_{n t}$ to be zero.

$$
\begin{aligned}
\varkappa_{c} & =-\left[\left(\left(A_{16}^{\prime} A_{26}^{\prime}-A_{12}^{\prime} A_{66}^{\prime}\right) B_{11}^{\prime}+\left(A_{11}^{\prime} A_{66}^{\prime}-A_{16}^{\prime 2}\right) B_{12}^{\prime}+\left(A_{12}^{\prime} A_{16}^{\prime}\right.\right.\right. \\
& \left.\left.-A_{11}^{\prime} A_{26}^{\prime}\right) B_{16}^{\prime}\right)\left(\left(A_{12}^{\prime} A_{26}^{\prime}-A_{16}^{\prime} A_{22}^{\prime}\right) N_{n}^{T}+\left(A_{11}^{\prime} A_{22}^{\prime}-A_{12}^{\prime 2}\right) B_{16}^{\prime 2}\right. \\
& \left.+\left(A_{12}^{\prime} A_{16}^{\prime}-A_{11}^{\prime} A_{23}\right) N_{t}^{T}+\left(A_{11}^{\prime} A_{22}^{\prime}-A_{12}^{\prime 2}\right) N_{n t}^{T}\right)+\left(A_{16}^{\prime 2} A_{22}^{\prime}\right. \\
& \left.-2 A_{12}^{\prime} A_{16}^{\prime} A_{26}^{\prime}+A_{11}^{\prime} A_{26}^{\prime 2}+A_{12}^{\prime 2}+A_{12}^{\prime 2} A_{66}^{\prime}-A_{11}^{\prime} A_{22}^{\prime} A_{66}^{\prime}\right)\left(\left(A_{12}^{\prime} A_{16}^{\prime}\right.\right. \\
& \left.\left.\left.-A_{11}^{\prime} A_{26}^{\prime}\right) M_{n}^{T}+\left(A_{26}^{\prime} B_{11}^{\prime}-A_{16}^{\prime} B_{12}^{\prime}\right) N_{n}^{T}+\left(A_{11}^{\prime} B_{12}^{\prime}-A_{12}^{\prime} B_{11}^{\prime}\right) N_{t}^{T}\right)\right] \\
& +\left[( A _ { 1 1 } ^ { \prime } A _ { 2 6 } ^ { \prime } - A _ { 1 2 } ^ { \prime } A _ { 2 6 } ^ { \prime } ) \left(\left(A_{22}^{\prime} A_{66}^{\prime}-A_{26}^{\prime 2}\right) B_{11}^{\prime 2}\right.\right. \\
& +2\left(A_{16}^{\prime} A_{26}^{\prime}-A_{12}^{\prime} A_{66}^{\prime}\right) B_{11}^{\prime} B_{12}^{\prime}+\left(A_{11}^{\prime} A_{66}^{\prime}-A_{16}^{\prime 2}\right) B_{12}^{\prime 2} \\
& +2\left(A_{12}^{\prime} A_{26}^{\prime}-A_{16}^{\prime} A_{22}^{\prime}\right) B_{11}^{\prime} B_{16}^{\prime}+2\left(A_{12}^{\prime} A_{16}^{\prime}-A_{11}^{\prime} A_{26}^{\prime}\right) B_{12}^{\prime} B_{16}^{\prime} \\
& +\left(A_{11}^{\prime} A_{22}^{\prime}-A_{12}^{\prime 2}\right) B_{16}^{\prime 2}+\left(A_{16}^{\prime 2} A_{22}^{\prime}-2 A_{12}^{\prime} A_{16}^{\prime} A_{26}^{\prime}+A_{11}^{\prime} A_{26}^{\prime 2}\right. \\
& \left.\left.\left.+A_{12}^{\prime 2} A_{66}^{\prime}-A_{11}^{\prime} A_{22}^{\prime} A_{33}^{\prime}\right) D_{11}^{\prime}\right)\right]
\end{aligned}
$$


The $N_{n}^{T}, N_{t}^{T}$ and $N_{n t}^{T}$ are the thermal force resultants per unit width, $M_{n}^{T}$ is the thermal moment resultant per unit width along the $n$-direction and $A_{i j}^{\prime}, B_{i j}^{\prime}$ and $D_{i j}^{\prime}$ are the laminate stiffnesses of the $[\theta+\alpha / \theta+\alpha]$ laminate $[15,16]$.

Again for large panels the curvature $\varkappa_{\mathrm{c}}$ resulting from Equation (19) is exactly similar to the asymptotic value of the curvatures obtained by using the theory presented above. Although Equation (19) is quite lengthy, it is still relatively simple to use instead of the approaches discussed so far.

\section{EXPERIMENTAL RESULTS}

Several unsymmetric laminates were produced for experimental determination of curvatures and comparison with the various theoretical models. The laminates were made by stacking unidirectional prepreg material. This $0.18 \mathrm{~mm}$ thick prepreg material was produced by Ten Cate Advanced Composites bv and consisted of glass-fibre reinforced PEI (polyetherimide, Ultem 1000 from General Electric Plastics). PEI is an amorphous thermoplastic and has a glass-transition temperature $\left(T_{g}\right)$ of $215^{\circ} \mathrm{C}$. The laminates were cured hot at $310^{\circ} \mathrm{C}$ and afterwards cooled to a room temperature of $21^{\circ} \mathrm{C}$ with a cooling rate of $0.0644\left({ }^{\circ} \mathrm{C} / \mathrm{s}\right)$.

The fibre volume fraction of the laminates produced is $V_{f}=0.55$ and the mechanical and thermal material properties established by measurements are presented in Table 1. The mechanical properties have been measured based on the ISO 527 Standard and the linear thermal expansion coefficients were measured according to methods recommended by the Measurement Group [19] in the range from $20^{\circ} \mathrm{C}$ to $180^{\circ} \mathrm{C}$ using large grid strain gauges. The desired temperatures were obtained in a circulation hot air oven.

A mould was designed which did not allow flow of the polymer at high temperature, to ensure that the fibres remained in position during curing and thus guaranteed good quality laminates with straight fibres. The initial dimensions of the laminates produced after trimming off the edges were $400 \times 400 \mathrm{~mm}^{2}$. After measuring the curvatures, successively smaller plates were cut from the $400 \mathrm{~mm}$ square laminate such that the centre point of all the panels was the same. The curvatures were established directly after moulding by measuring several positions on straight lines within the laminates. Contactless measurements of outof-plane deflections were made to ensure that the measuring technique did not cause deflections of the thin laminates; this made sure that the out-of-plane deflections could be established with an accuracy of $0.05(\mathrm{~mm})$. The correspond-

Table 1. Mechanical properties of glass/PEI.

$\begin{array}{rlrl}E_{1}=43.1 & (\mathrm{GPa}) \\ E_{2}=14.3 & (\mathrm{GPa}) \\ V_{12}=0.270 & & \\ G_{12}=5.5 & (\mathrm{GPa}) \\ \alpha_{1}=7.49 \times 10^{-6} & (1 / K) \\ \alpha_{2}=2.59 \times 10^{-5} & & (1 / K)\end{array}$




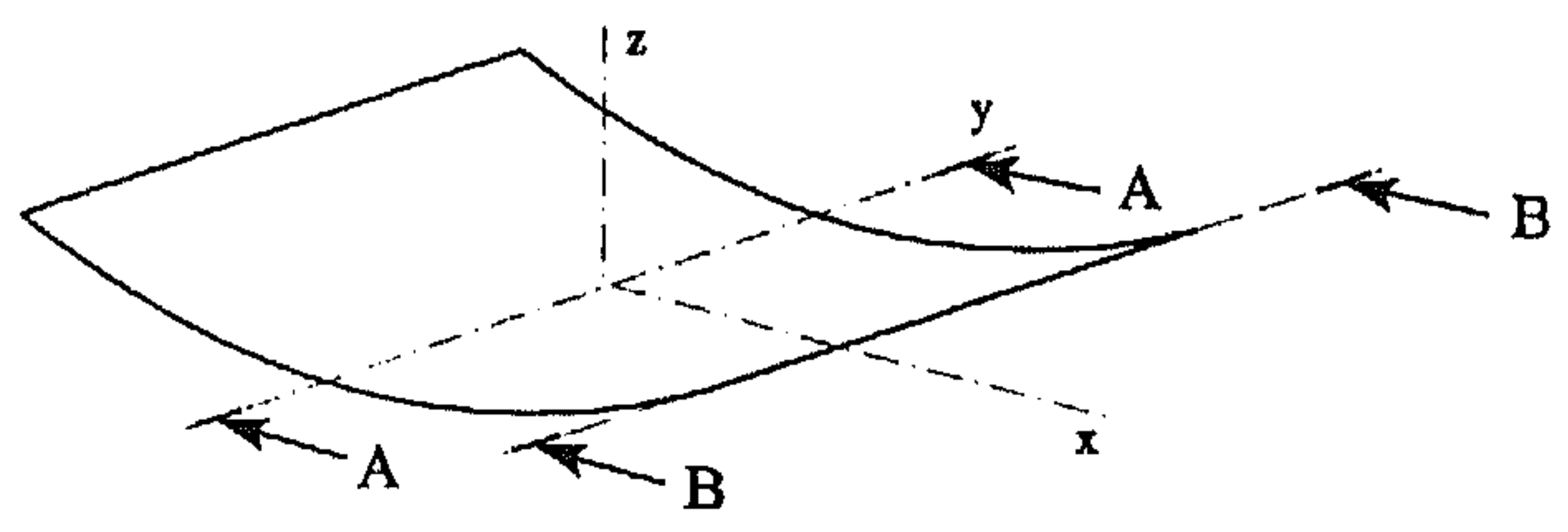

A-A

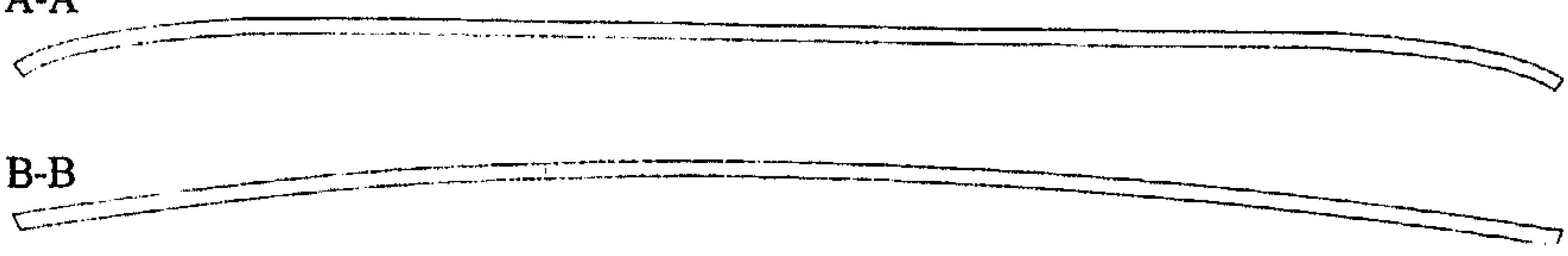

Figure 11. Observed free-edge effects on $200 \mathrm{~mm} \times 200 \mathrm{~mm}$ [0/90] laminate.

ing $x$ and $y$ coordinates in the horizontal plane were determined with an accuracy of $0.0025(\mathrm{~mm})$.

During measurements, it was observed that the laminates produced were not perfectly cylindrical. The laminates showed some free-edge effects. Figure 11 shows 2 cross sections of a cross-ply laminate where the curvature should be zero, sections A-A and B-B are at the symmetry axis of the plate and at one edge of the plate respectively and give an indication of the offset from a non-curved (straight) section. The curvature along A-A is globally zero but some nonzero curvature is present at the edge. The curvature along B-B is definitely nonzero. Similar behaviour is also present in an angle-ply laminate. Figure 12 shows two cross sections, one along the $t$ axis and the other parallel to the $t$ axis and near

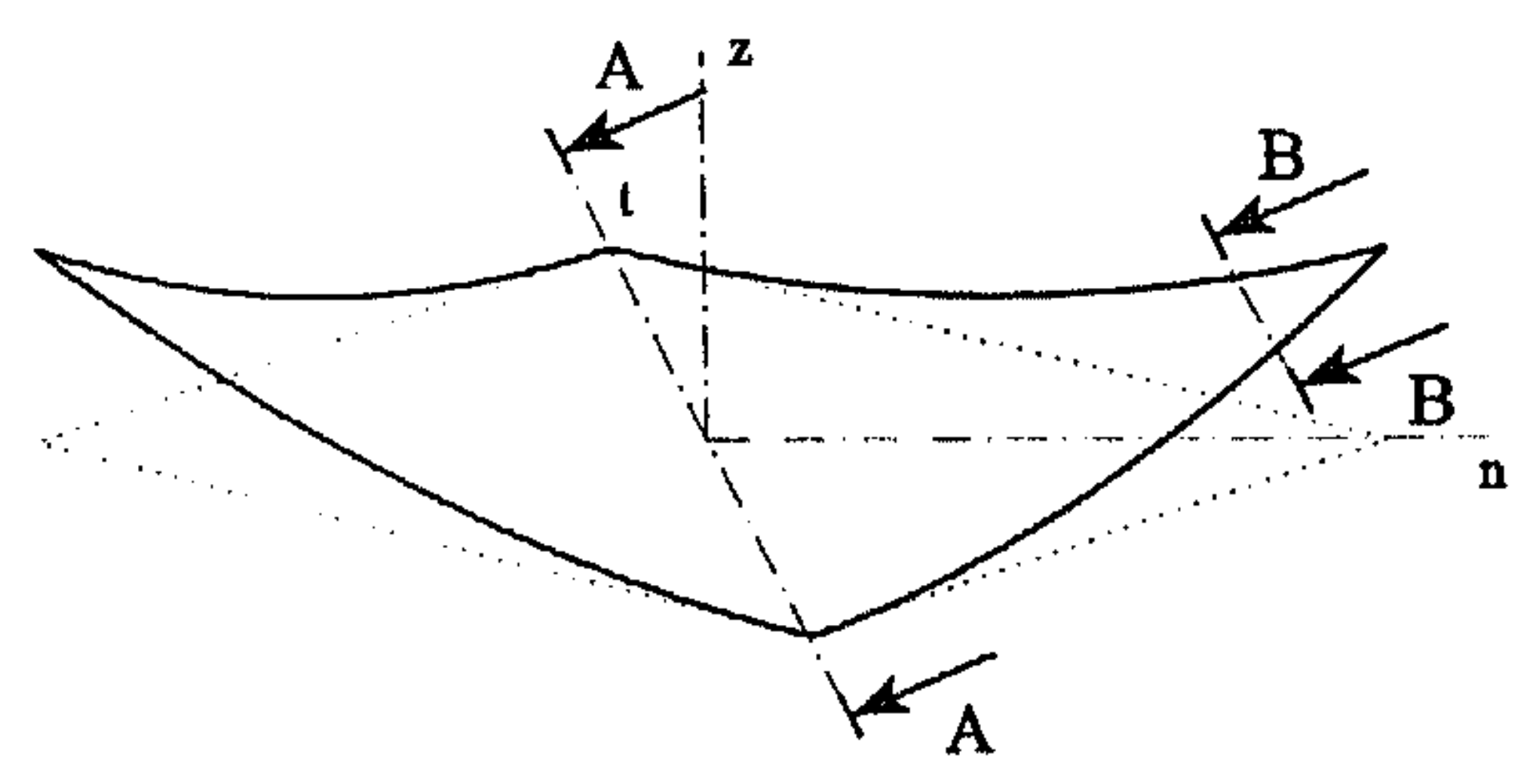

A-A

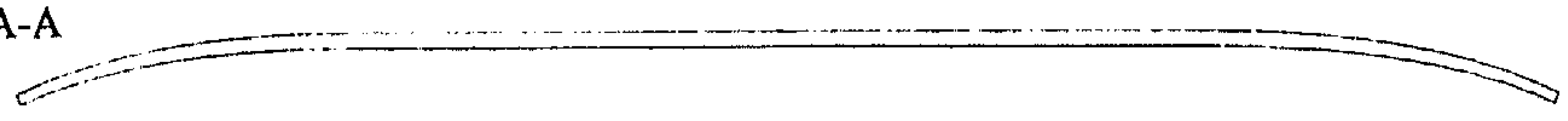

B-B

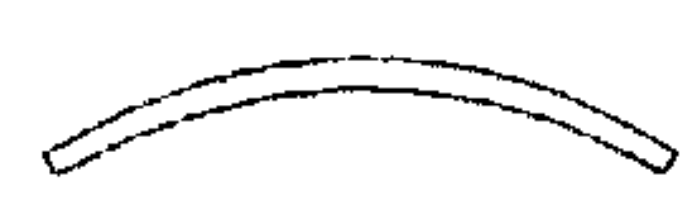

Figure 12. Observed free-edge effects on a $200 \mathrm{~mm} \times 200 \mathrm{~mm}[-30 / 30]$ laminate. 
a corner, where the curvature should be zero in an angle-ply laminate with cylindrical shape. Again A-A and B-B give an indication of the offset from a noncurved section. The free-edge effects are not taken into consideration in this paper.

In Figure 13 the theoretically predicted and experimentally measured curvatures are compared for a $\left[\mathrm{O}_{2} / 90_{2}\right]$ cross-ply laminate. To obtain the theoretical predictions the displacement field is approximated by function (9). Above the bifurcation point $x_{1}$ and $x_{2}$ are the curvatures of the first (principal curvatures $\left.\left[x_{1}, 0\right]\right)$ and second (principal curvatures $\left.\left[0, x_{2}\right]\right)$ cylindrical shape respectively. After release from the mould there is a slight preference for one cylindrical shape, which shows the largest curvatures $\left(x_{1}\right)$. Below the bifurcation point $x_{1}$ and $\varkappa_{2}$ are the curvatures of the saddle shape. Again one curvature is larger, along the axis where also the cylindrical deformation was the largest.

It is worth noting that the result given by the classical lamination theory is the one corresponding to the side length of zero. Bifurcation is predicted to occur at a side length of $57 \mathrm{~mm}$, which is close to what experiments indicate. The experimental results agree reasonably well with the predicted curvatures (on average the predicted curvature values of the cylindrical deformations are $15 \%$ larger than the experimentally measured values).

The results for a $\left[30_{2} /-30_{2}\right]$ angle-ply laminate are presented in Figure 14 . The curvatures shown are curvatures along the $n$ - and $t$-axis of the principal coordinate system $n-t-z$ and the theoretical predictions are obtained by using displacement functions (15) and (16). Above the bifurcation point $x_{1}$ and $x_{2}$ are the curvatures of the first and second cylindrical shape. Again there is a slight preference for one of the two cylindrical shapes (this shape shows the largest curvature).

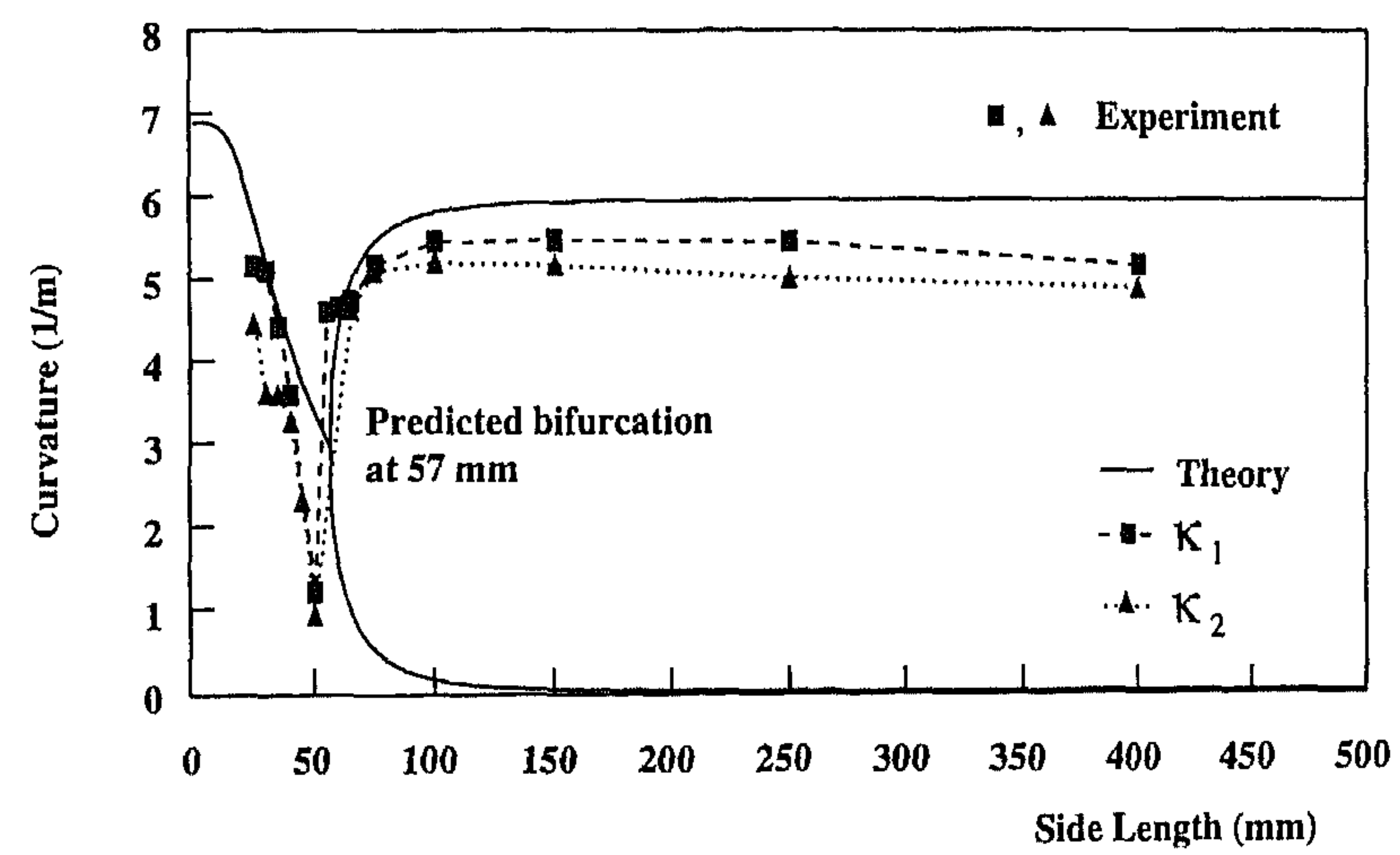

Figure 13. Predicted and experimentally measured curvatures for a $\left[\mathrm{O}_{2} / 9 \mathrm{O}_{2}\right]$ glass/PEI laminate (ply thickness $h_{p}=0.18 \mathrm{~mm}$ ). 


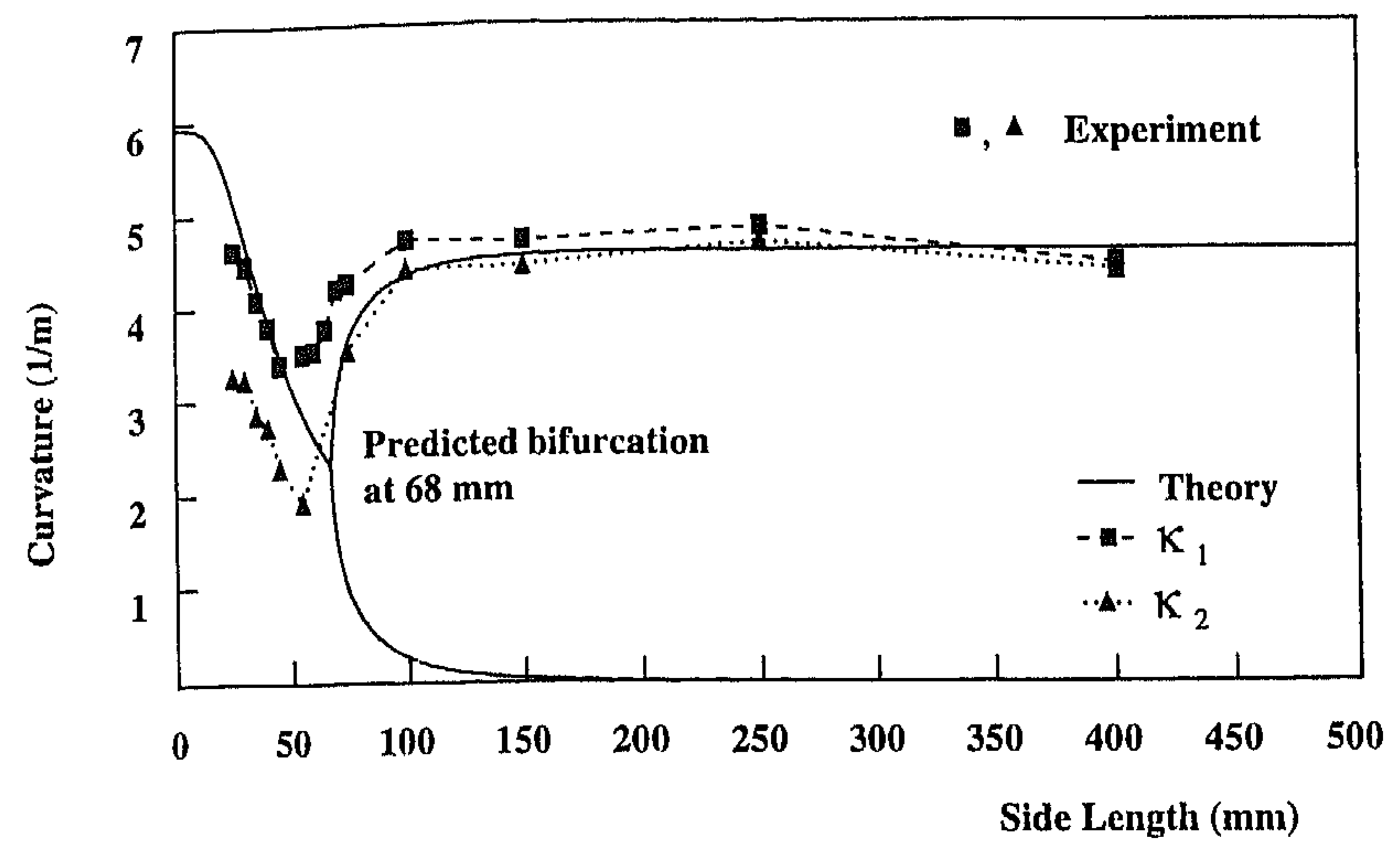

Figure 14. Predicted and experimentally measured curvatures for a $\left[3 \mathrm{O}_{2} /-3 \mathrm{O}_{2}\right]$ glass/PEI laminate (ply thickness $h_{p}=0.18 \mathrm{~min}$ ).

Below the bifurcation point are $\varkappa_{1}$ and $\varkappa_{2}$, the curvatures of the twisted shape. As might be expected the curvatures of a $\left[30_{2} /-30_{2}\right]$ angle-ply laminate are lower than the curvatures of the cross-ply laminate shown in Figure 14, because the mismatch in thermal shrinkage across the thickness is proportional to the difference in orientation between adjacent plies.

On average the predicted curvature values of the cylindrical deformations are only $2.7 \%$ lower than the experimentally measured values. Both qualitatively and quantitatively the curvatures of the unsymmetric laminates are very well predicted.

Although the resemblance between theory and experiment is good, especially for the angle-ply laminates, there still are several causes for differences between theory and experiment. These causes for differences are outlined in more detail in the following sections.

\subsection{Effect of Thermal and Mechanical Properties Used}

The theoretically predicted curvatures are sensitive to the thermal and mechanical properties used. This is particularly important for the transverse thermal expansion coefficient $\alpha_{2}$, which is difficult to determine. Normal Thermomechanical Analysis (TMA) techniques (according to the ASTM E831-86 standard for linear thermal expansion) use very small specimens, therefore as experienced, local inhomogeneities can have a large influence on the results. This is the reason why large grid strain gages [19] were used for measuring the expansion coefficients. The obtained values were consistent. The values of the linear thermal expansion coefficients were checked by also measuring them with a specially de- 
signed dilatometer setup for large specimens. The results were quite similar to those measured by means of strain gages.

In Figure 15 the effect is shown when a 10 percent higher transverse thermal expansion coefficient than the measured value presented in Table 1 is used in the calculations. Bifurcation occurs at a smaller laminate size $(53 \mathrm{~mm}$ instead of 57 $\mathrm{mm}$ ) and the predicted curvatures are significantly larger. The asymptotic value of the cylindrical deformations increases by 14.1 percent.

Fibre/polymer composites often behave differently under tension and compression. The compression moduli are often not as great as the tensile moduli [13], Internal stresses result in out-of-plane (bending) deformations of unsymmetric laminates. Therefore, some layers will be in compression and some will be in tension. For a four layer $\left[\mathrm{O}_{2} / 90_{2}\right]$ laminate which shows in theory perfect cylindrical deformation, the stresses along the fibres and at right angles to the fibres are shown in Figure 16. The stress profiles are calculated by taking geometric nonlinear behaviour into consideration. Therefore, force equilibrium over the thickness is no longer fulfilled (shear stresses are present).

Along the fibre direction the most layers are in compression (except the top of the top layer) and at right angles to the fibre direction the most layers are in tension (except the bottom of the bottom layer). To determine the moduli $E_{1}$ and $E_{2}$ in compression, compression tests were carried out (according to the ASTM D 3410-87 standard). The results are shown in Table 2.

Only $E_{2}$ is significantly lower than the value measured in tension. Because at right angles to the fibres all layers are completely or almost completely in tension and $E_{\text {1ransion }} \approx E_{\text {1compr. }}$, the Young's moduli used will be about correct. On top of this, analysis shows that the curvatures are not very sensitive for changes in Young's moduli. Therefore, bimodulus behaviour $\left(E_{1 \text { tension }} \neq E_{1 \text { compr. }}\right)$ will

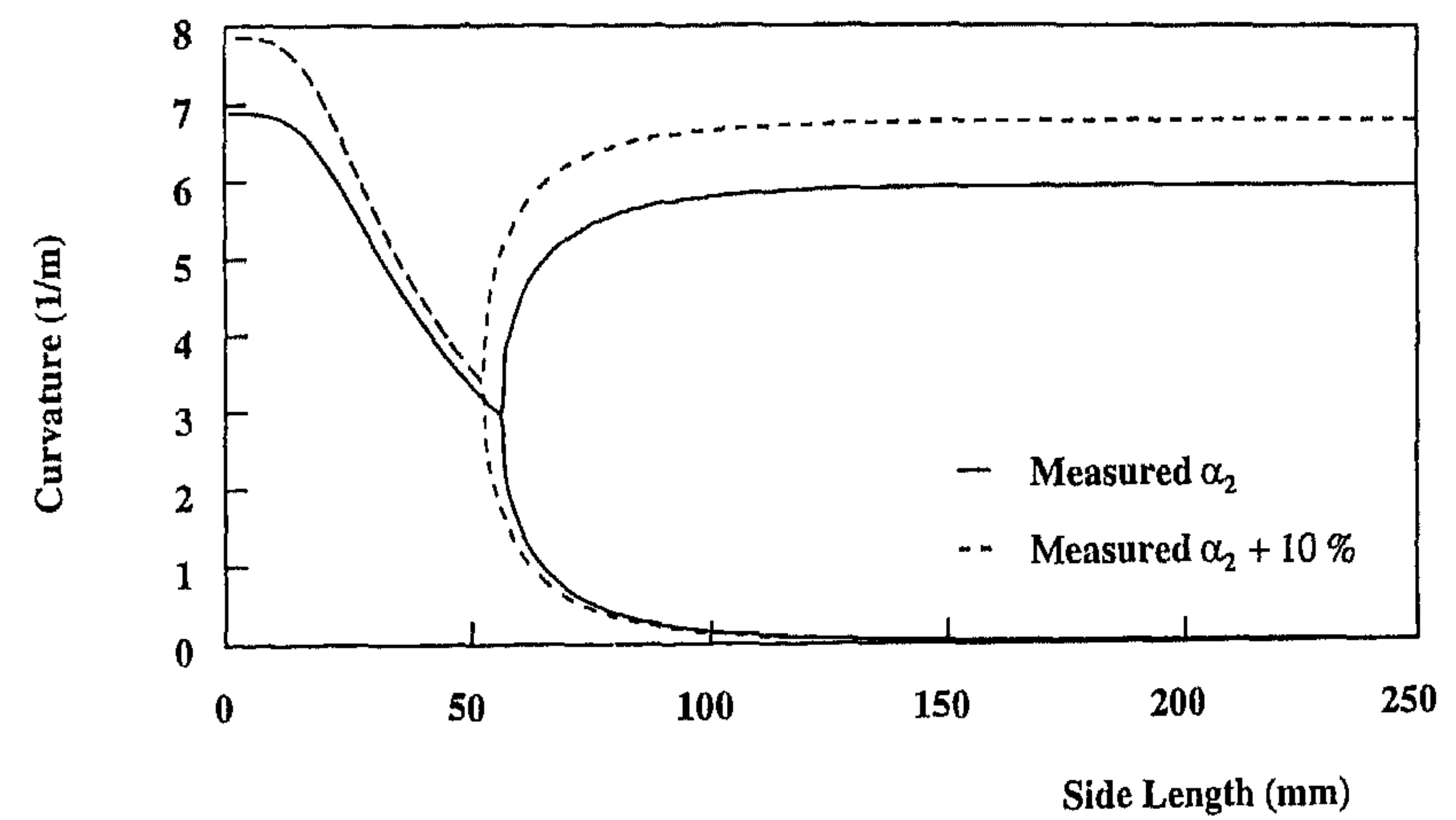

Flgure 15. The effect of a 10 percent change in $\alpha_{2}$ on the predicted curvatures for a $\left[\mathrm{O}_{2} / 9 \mathrm{O}_{2}\right]$ glass/PEI laminate. 


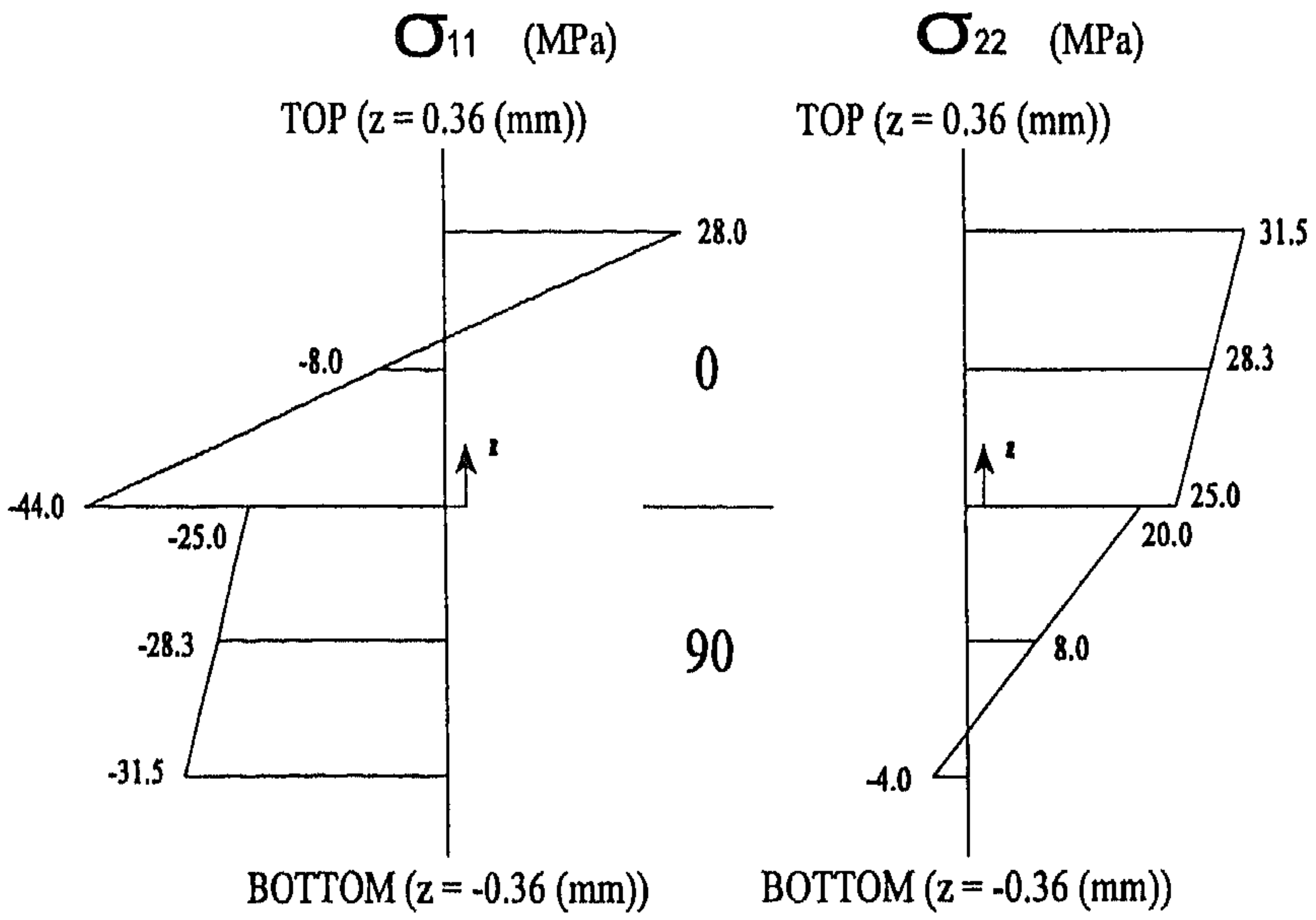

Figure 16. Through the thickness stress profiles of a large $\left[\mathrm{O}_{2} / 9 \mathrm{O}_{2}\right]$ glass/PEI laminate.

not contribute significantly to the difference between predicted and measured curvatures.

The properties of fibre-polymer laminates are temperature dependent, especially in the temperature range around $T_{8}$. The presented theory assumes that the thermoelastic properties are insensitive to temperature changes, which may also cause differences between measured and predicted curvatures.

In general exact (temperature-dependent) values of the thermal and mechanical properties are important for a good quantitative prediction of the curvatures in relation to the side length. In particular the value for the transverse thermal expansion coefficient $\alpha_{2}$ is essential.

\subsection{Effect of Manufacturing Parameters}

The prepreg, which was used to produce the laminates, did not have an ideally symmetric fibre distribution. Due to the manufacturing technique, one side was

Table 2. Young's modull of glass/PEI.

\begin{tabular}{ll}
\hline$E_{1 \text { compr. }}=42.5$ & $(\mathrm{GPa})$ \\
$E_{1 \text { tension }}=43.1$ & $(\mathrm{GPa})$ \\
$E_{2 \text { compr. }}=10.7$ & $(\mathrm{GPa})$ \\
$E_{2 \text { tension }}=14.3$ & $(\mathrm{GPa})$ \\
\hline
\end{tabular}


fibre rich and the other side was resin rich, so that the fibres were not in the central plane of the prepreg. Consequently, laminates produced with this prepreg did not have an ideal uniform fibre distribution as assumed in the theory. Because of the nonsymmetry in the prepreg, it was possible to construct four layer balanced unsymmetric laminates with four different lay-ups. (Balanced in this case means that the two cylindrical shapes, which could be obtained, showed more or less identical curvatures.) Each lay-up resulted in different curvatures. Photographs of the cross sections of two different lay-ups are shown in Figure 17; the difference in fibre distribution is quite obvious.

Different lay-up sequences result in different curvatures. For large panel sizes differences up to 10 percent could be obtained. Laminates with the fibres as far as possible away from the midplane showed the smallest curvatures, because of the larger second moment of area. For the laminates produced, the theory which assumes uniform fibre distribution within the layers of the laminate needs some adjustments.

\subsection{Effect of Stress-Free Temperature Used}

Theoretically, it was assumed that the temperature at which thermal stress build-up initiates equals the glass transition temperature of the resin. However, Wang et al. [12] showed for thermoset composites that the stress-free temperature can be quite different from the glass transition temperature. For the glass/ PEI material used, this was checked by producing laminates with an adjusted cooling procedure. By stopping the cooling procedure for some time at $T_{8}$, stresses which could have been built up at higher temperatures had time to relax. Relaxation of these stresses should result in a change in curvature. However, the laminates produced with the adjusted cooling procedure showed no significant difference in curvature. Therefore, it was concluded that the thermal stress build-up temperature is close to $T_{g}$ as assumed. Kim et al. [11] concluded the same for a graphite/polyimide composite. Like polyetherimide (PEI), polyimide (PI) is an amorphous thermoplastic resin.

Still, a stress-free temperature which is slightly different than $T_{8}$, will have an effect on the theoretically predicted curvatures. Figure 18 shows the influence on the theoretically predicted curvatures of a stress-free temperature $\left(T_{c}\right)$ which is $10^{\circ} \mathrm{C}$ higher than assumed $\left(=T_{s}+10^{\circ} \mathrm{C}\right)$. This (already large) change in thermal stress build-up temperature, results in a $5.1 \%$ increase in the asymptotic value of the cylindrical curvatures.

\section{CONCLUSIONS}

In order to make full use of the possibility to produce curved panels from flat moulds, it is important that the cured shapes of unsymmetric laminates can be well predicted. Theoretical models to describe these shapes have been reviewed. The models based on the minimization of the total potential energy, use an out-ofplane displacement relation, which is a third order approximation of the actual shapes. It is shown that this third order approximation is accurate enough to obtain reliable results for the cured shapes. It is also shown that it is important 







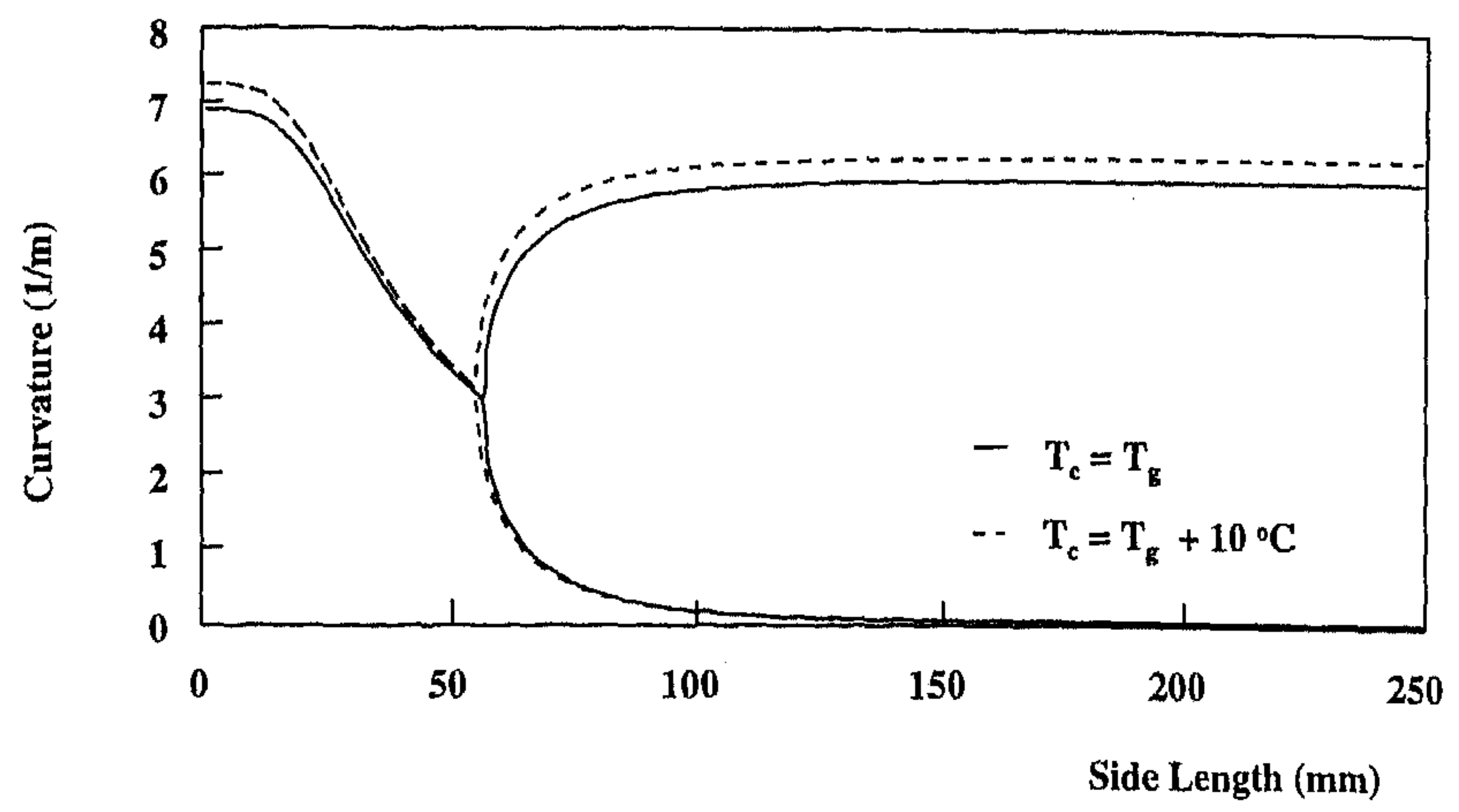

Figure 18. The effect of a $10^{\circ} \mathrm{C}$ change in $\mathrm{T}_{0}$ on the predicted curvatures for a $\left[\mathrm{O}_{2} / 9 \mathrm{O}_{2}\right]$ glass/PEl laminate.

to take the shear strain correctly into account, even for a cross-ply laminate (according to the C.L.T. there is no shear strain in a cross-ply laminate).

For cross-ply laminates theoretically predicted curvatures, obtained with the model based on the minimization of the total potential energy and which includes shear strain, we compared with results obtained by using finite element analysis. The results agree quite well. Therefore, it is concluded that with this model reliable theoretical results can be obtained for the cured shapes of unsymmetric cross-ply laminates.

The relatively simple method based on the C.L.T. for calculating the curvature of the (perfectly) cylindrical shapes of large unsymmetric laminates give similar results than the methods based on the minimization of the total potential energy.

The experimental work that has been conducted shows that the influence of the side length on the curvature is very well predicted, especially for square angleply laminates. For square cross-ply laminates the predicted values of the curvatures are somewhat high. For a quantitative good prediction exact values of the thermal and mechanical properties are important. In particular, the value for the transverse thermal expansion coefficient $\alpha_{2}$ is essential; a slight change in $\alpha_{2}$ has a significant influence on the predicted curvatures. Further, the theoretical model assumes a uniform fibre distribution within the layers. Because of nonsymmetry in the prepreg, the laminates produced did not have an ideal uniform fibre distribution as assumed in theory.

The main conclusion for the designer who is interested in producing curved panels with a flat mould is that the method based on the classical lamination theory gives a cheap and good approximation of the curvature developed by large unsymmetric cross-ply and angle-ply and angle-ply laminates. One of the numerous software packages that follow the C.L.T. can be used, simply by setting $x_{x}$ or 
$x_{y}$ (and $x_{x y}$ in case of angle-ply laminates) to be zero in the principal curvature coordinate system.

\section{ACKNOWLEDGEMENTS}

The authors wish to thank $\mathrm{H}$. J. M. Geijselaers for his valuable remarks made with respect to the work reported and Ten Cate Advanced Composites bv for providing the prepreg material for the experimental part of the work.

\section{REFERENCES}

1. Hyer, M. W. 1981. "Some Observations on the Cured Shape of Thin Unsymmetric Laminates," J. of Comp. Materials, 15:175-194.

2. Hyer, M. W. 1981. "Calculation of the Room-Temperature Shapes of Unsymmetric Laminates," J. of Comp. Materials, 15:296-310.

3. Hyer, M. W. 1982. "The Room-Temperature Shapes of Four-Layer Unsymmetric Cross-Ply Laminates," J. of Comp. Materials, 16:318-340.

4. Jun, W. J. and C. S. Hong. 1990. "Effect of Residual Shear Strain on the Cured Shape of Unsymmetric Cross-Ply Thin Laminates," Comp. Science \& Technology, 38:55-67.

5. Jun, W. J. and C. S. Hong. 1992. "Cured Shape of Unsymmetric Laminates with Arbitrary Lay-Up Angles," J. of Reinforced Plastics and Comp., II:1352-1366.

6. Hahn, H. T. and D. G. Hwang. 1981. "Residual Stresses and their Effect in Composite Laminates," Proc. of NCKU/AAS Int. Symposium on Eng. Sciences and Mechanics, Taiwan.

7. Hahn, H. T. 1981. "Warping of Unsymmetric Cross-Ply Graphite/Epoxy Laminates," Composite Review, 3:114-117.

8. White, S. R. and H. T. Hahn. 1992. "Process Modeling of Composite Materials: Residual Stress Development During Cure. Part I. Model Formulations," J. of Comp. Materials, 26:2402-2422.

9. White, S. R. and H. T. Hahn. 1992. "Process Modeling of Composite Materials: Residual Stress Development During Cure. Part II. Experimental Validation," J. of Comp. Materials, 26:2423-2454.

10. White, S. R. and H. T. Hahn. 1993. "Cure Cycle Optimization for the Reduction of ProcessingInduced Residual Stresses in Composite Materials," J. of Comp. Materials. 27:1352-1378.

11. Kim, K-S, H. T. Hahn and R. B. Croman. 1989. "The Effect of Cooling Rate on Residual Stress in a Thermoplastic Composite," J. of Comp. Technology \& Research, 11(2):47-52.

12. Wang, H-B., S-J Li and T-Y Yu. 1993. "On the Thermal Behavior and Mechanism of Residual Microstress Build-Up at the Matrix Interface in Filled Epoxy Resins," Polymer Engineering and Science, 33(8):474-479.

13. Mrse, A. and M. R. Piggott. 1990. "Relation Between Fibre Divagation and Compressive Properties of Fibre Composites," Proc. of 35th Int. SAMPE Symposium.

14. Warnet, L. 1992. "Cured Shapes of Thin Laminated Plates," University of Twente, MSc thesis.

15. Powell, P. C. 1994. "Engineering with Fibre/Polymer Composites," Chapman \& Hall.

16. Tsai, S. W. and H. T. Hahn. 1980. "Introduction to Composite Materials," Lancaster, PA: Technomic Publishing Co.

17. Timoshenko, S. P. and S. Woinowsky-Krieger. 1964, "Theory of Plates \& Shells," McGraw-Hill.

18. Fung, Y. C. 1965. "Foundations of Solid Mechanics," Prentice-Hall.

19. Measurements Group, Inc. 1986. "Measurement of Thermal Expansion Coefficient Using Strain Gages," Tech. Note TN-513. 